\begin{abstract}
SEXTON, CHRISTOPHER. Vectorization of Gridded Urban Land Use Data. (Under the direction of Dr. Benjamin Watson.)

In the digital entertainment industry, cities are one of the largest artifacts modeled by artists. One alternative to modeling an entire city by hand is to use an urban simulation. Often, those simulations use a gridded terrain representation. Translating gridded simulation results into a more continuous, realistic representation can often be difficult. Our vectorization process transforms gridded urban land use data into a representation that mimics what might be seen in GIS or online mapping tools.

Our method consists of three major phases. In the first phase, the raster data is is analyzed and the transportation layer is abstracted and filtered. Next, the city blocks are constructed from the raster data. Third, the blocks are subdivided and land use and density are assigned to each constructed parcel. The result is scored and compared with the output from the simulation to match location, sizes, and proportions.
\end{abstract}




\title{
Vectorization of Gridded Urban Land Use Data
}

\author{
by
}

\section{Christopher G. Sexton}

A thesis submitted to the Graduate Faculty of

North Carolina State University

in partial fulfillment of the

requirements for the Degree of

Master of Science

\section{Computer Science}

\author{
Raleigh, North Carolina
}

2007

Approved By:

Dr. Christopher Healey

Kofi Boone

Dr. Benjamin Watson

Chair of Advisory Committee 


\section{Biography}

Chris Sexton was born in Akron, Ohio, but spent most of his childhood in the mountains of North Carolina, in a small town named Sparta. Chris graduated Summa Cum Laude from North Carolina State University in December 2005 with a B.S. in Computer Science. He was also named a Valedictorian of the Fall 2005 graduating class. He expects to receive his M.S. in Computer Science from North Carolina State University in December 2007. 


\section{Acknowledgements}

I would like to thank my advisor, Dr. Benjamin Watson, for his continued support and

guidance throughout my graduate career. I would also like to thank Dr. Christopher Healey and Kofi Boone for serving on my thesis committee. 


\section{Contents}

List of Figures $\quad$ vi

1 Introduction 1

2 Related Work 3

2.1 Pioneering Procedural Modeling Work . . . . . . . . . . . . . . . . 3

2.2 Generation of 3D Structures . . . . . . . . . . . . . . . 4

2.2.1 3D Model Synthesis . . . . . . . . . . . . . . . . 4

2.2 .2 3D Architectural Synthesis . . . . . . . . . . . . 5

2.3 Generation of 2D Patterns . . . . . . . . . . . . . . . 7

2.3.1 Texture Synthesis . . . . . . . . . . . . . . . . . 7

2.3 .2 Terrain Synthesis . . . . . . . . . . . . . . . . 8

2.3 .3 Urban Synthesis . . . . . . . . . . . . . . . . . 8

2.4 Vectorizing $2 \mathrm{D}$ Patterns $\ldots \ldots \ldots \ldots \ldots \ldots$

3 Framework $\quad 11$

3.1 Initial Data Preprocessing . . . . . . . . . . . . . . . . . . . 11

3.1 .1 Input Data Loading . . . . . . . . . . . . . . . . . . . . 11

3.1 .2 Raw Block Generation . . . . . . . . . . . . . . . . . . . 11

3.1.3 Extraneous Intersection Patch Cleaning . . . . . . . . . . . . . . . . 12

3.1.4 Road Graph Generation . . . . . . . . . . . . . . . . . . . . 13

3.1.5 Parcel Data Loading . . . . . . . . . . . . . . . . . 15

3.2 Road Correction . . . . . . . . . . . . . . . . . . 16

3.2 .1 Intersection Straightening . . . . . . . . . . . . . . . 16

3.2 .2 Road Smoothing . . . . . . . . . . . . . . . . . . . 17

3.2.3 Primary and Secondary Connection . . . . . . . . . . . . . 17

3.2 .4 Cycle Removal . . . . . . . . . . . . . . . . . . . . . 18

3.2 .5 Intersection Simplification . . . . . . . . . . . . . . 18

3.2 .6 Curve Smoothing . . . . . . . . . . . . . . . . . . . . . . . 19

3.3 Road Geometry Creation . . . . . . . . . . . . . . . . . 20

3.4 Vectorized Block Development . . . . . . . . . . . . . . . . 21

3.4.1 Matching of Simulation Blocks to Generated Blocks . . . . . . . . . 23

3.4.2 Generation of Polygonal Spines . . . . . . . . . . . . 23 
3.4.3 Subdivision of Blocks . . . . . . . . . . . . . . . . . 24

4 Implementation $\quad 32$

5 Results 33

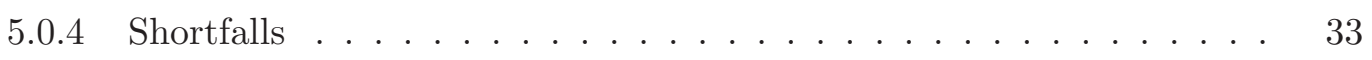

5.0 .5 Moving Forward . . . . . . . . . . . . . . . . . . . . 33

5.0.6 Examples of Vectorization . . . . . . . . . . . . . . . 34

$\begin{array}{ll}\text { Bibliography } & 44\end{array}$ 


\section{List of Figures}

2.1 Example of building texture correctness from Dorsey et. al [21] . . . . . . 6

2.2 Example of road generation from Parish and Muller [29] . . . . . . . . . 9

2.3 Extraction of segmented road network by Mena $[24] \ldots \ldots$

3.1 View of initial data from simulation . . . . . . . . . . . . . . 12

3.2 Extraneous road patch removal . . . . . . . . . . . . . . . . 13

3.3 Graph connectivity before cycle elimination . . . . . . . . . . . . . . . 14

3.4 Graph connectivity after cycle elimination . . . . . . . . . . . . . . . 14

3.5 Cluster of loops that don't represent an intersection . . . . . . . . . . . . 14

3.6 Data ready for manipulation and processing . . . . . . . . . . . 16

3.7 Intersection straightening (Left: before, Right: after) . . . . . . . . . . . 17

3.8 Road smoothing and intersection straightening . . . . . . . . . . . 18

3.9 After connecting secondary and primary . . . . . . . . . . . . . 19

3.10 Short cycle to be removed (Left: before, Right: after) . . . . . . . . . . . 19

3.11 Increased point count in curves preserves features . . . . . . . . . . . . . . 20

3.12 Road geometry with road edges highlighted . . . . . . . . . . . . 21

3.13 Example of block generated from water line . . . . . . . . . . . . . 22

3.14 Example of block generated from external boundary lines . . . . . . . . . 23

3.15 Spine generation: Top, binary. Bottom, polygonal. . . . . . . . . . 25

3.16 Example of generated spines for blocks . . . . . . . . . . . . . 26

3.17 Example of land use assignment: (yellow $=$ residential, blue $=$ industrial, red = commercial, green $=$ park $\ldots \ldots \ldots \ldots . \ldots \ldots 29$

3.18 Example of density (dark is higher density) . . . . . . . . . . . 31

5.1 Raw simulation data: Data set $1 \ldots \ldots \ldots$. . . . . . . . . . 35

5.2 Land use assignments: Data set 1 . . . . . . . . . . . . . . . . 36

5.3 Population density visualization: Data set $1 \ldots \ldots \ldots$. . . . . . . . . 37

5.4 Raw simulation data: Data set $2 \ldots \ldots \ldots \ldots$

5.5 Land use assignments: Data set 2 . . . . . . . . . . . . . . . . 39

5.6 Population density visualization: Data set 2 . . . . . . . . . . . . 40

5.7 Raw simulation data: Data set $3 \ldots \ldots$. . . . . . . . . . . 41

5.8 Land use assignments: Data set $3 \ldots \ldots$. . . . . . . . . . . . . . . . . . . . . . . . . . . . . . .

5.9 Population density visualization: Data set $3 \ldots \ldots \ldots$. . . . . . . 43 


\section{Chapter 1}

\section{Introduction}

Generating urban content and models is one of the most challenging problems facing artists working in the digital entertainment and training industries. Urban synthesis is concerned with generating urban content in a unique, procedural way. Things such as building placement, zoning, roads, and actual building generation are all examples of urban synthesis. We currently have a simulation tool that assists in zoning and the location of buildings, however the simulation uses a grid with resolution adequate for simulation efficiency and accuracy, but far too coarse for entertainment and training applications. Our vectorization method automatically transforms gridded urban simulation data into a more continuous and realistic representation. A brief overview of the vectorization process follows.

Vectorization moves through three stages: building a topologically connected transportation network, extracting and identifying city blocks, and subdivision of blocks into parcels. We assume that the gridded input has at least four layers: one describing land use, one describing usage density, another describing ownership, and the last describing elevation. We also assume that roads are disjoint if they are not four-connected on the input grid (a grid element at location $(x, y)$ is only four-connected to the elements at locations $(x+1, y),(x-1, y),(x, y+1)$ and $(x, y-1))$.

We first create an undirected graph that represents the transportation network. We link graph nodes if the corresponding road grid cells are four-connected, then smooth the resulting network by averaging connected graph neighbors. We next connect primary and secondary road segments and eliminate overly short graph cycles. After additional smoothing, we define road curbs and intersections, and place circles on cul-de-sacs. 
Blocks in the city center are defined by surrounding curb loops. At some locations such as the edge of the city, blocks will be bounded not only by curbs, but also by parcel or water boundaries. We then identify oblong or "pierced" blocks that would benefit from internal spines used during subdivision. Oblong blocks have oriented bounding boxes with aspect ratios less than 1:2. "Pierced" blocks have a cul-de-sac in their boundary, and should have parcel boundaries radiating from the cul-de-sac circle into a spine. We generate spines using an image skeleton algorithm [44].

To create parcels of ownership and assign use and density, we first match each vectorized block to a simulated block. We then subdivide each block to produce the same number of parcels as the simulated block, using a constrained and randomized binary space partitioning. We assign parcel use and density with a similarly randomized and constrained process that references the matching simulation block. We generate 50 such parcelings, and retain only the parceling that matches the simulation most closely using a scoring method devised to determine how closely the assignment matches the output from the simulation. We then export the data to an ESRI shapefile that can be read by various GIS and modeling tools, as the end product of the vectorization and assignment process.

To understand why we are interested in this sort of data filtering and enhancement, it is important to have an overview of procedural modeling on the whole. We begin with a review of procedural modeling, then delve into more applicable subfields, such as texture and image synthesis, image vectorization, terrain synthesis, 3D model synthesis, and finally architectural synthesis. Then, we document our research and findings, detailing algorithms developed and showing results. 


\section{Chapter 2}

\section{Related Work}

\subsection{Pioneering Procedural Modeling Work}

Procedural modeling is a very generic term for many different techniques in computer graphics used to create models and textures from a series of rules. Currently, hardware is getting increasingly powerful which allows for more and more detailed models to be created and rendered. Unfortunately, as model complexity increases, the amount of storage needed to store models climbs and the amount of time needed to model complex objects increases. For example, in the movie King Kong, Weta Digital created over 90,000 3D buildings, constructed from over 22 million components [42]. Most of the city of New York was modeled procedurally, while artists focused their attention on the landmark buildings. This allowed digital artists to concentrate on the artistically important aspects of the city model, without having to be worried about the other 90,000 buildings that needed to be recreated for the film. For a good survey of procedural textures and modeling, see [10]. In our area of research, procedural modeling is used for urban synthesis. Things such as building placement, zoning, roads, building generation and building weathering are all examples of urban synthesis that could be implemented using procedural modeling.

Procedural modeling isn't a new idea. It has been used for everything from terrain generation to simulating patterns of nature. In 1983, Fournier [13] developed a new solution to approximate fractional Brownian motion to compute terrain models. By the use of a new algorithm, he was able to create varying levels of detail in a surface without increasing the storage used, as well as allowing for deformation of surfaces by only changing a few

parameters. In the late 1980s early 1990s, Prusinkiewicz [34] used Lindenmayer systems 
to model complex plant structures . Formalized L-systems are grammars that can be used to model complex plants, such as trees and flowers. In 1983, Reeves introduced the notion of particle systems [35], which are used for modeling fuzzy objects such as fire, water, clouds, and smoke. This use of stochastic processes was incredibly useful for the visual effects community, and later the gaming community. Perlin [30] created a set of stochastic algorithms used to create representations of clouds, fire, and many other naturalistic images using only a few functions and basic input. Reynolds [36] reapplied particle systems to model bird flocking, animal herding, and the schooling patterns of fish. The rules followed by these particles are incredibly simple, but can model complex processes without excessive computational overhead. Finally, Sims [37] created models of virtual creatures using genetic algorithms that evolve creatures well suited to the input environment.

\subsection{Generation of 3D Structures}

\subsubsection{D Model Synthesis}

\section{Geometry}

Texture synthesis techniques (see below) can synthesize not only 2D images, but also 3D models. Bhat et. al [3] add surface geometry to 3D models by extending 2D texture synthesis to operate on volumetric models. Lagae et al. [20] extend this idea to synthesize unique models. For example, their technique can synthesize unique terrain given a small input model. Cutler et. al [7] use a script-based approach to generate layered, solid models such as sculpture.

Although our simulation doesn't generate 3D objects, it does locate and lay out $3 \mathrm{D}$ buildings, given an input terrain. We anticipate combining our $2 \mathrm{D}$ simulation with $3 \mathrm{D}$ model synthesizers such as these to create interesting and realistic city models.

\section{Textures on Geometry}

Urban detail consists not only of the buildings themselves, but also the effect of weathering on those buildings. In 1999, Dorsey et. al [9] performed work on the modeling and rendering of weathered stone. They presented a method of rendering changes in shape and surface appearance of stone to mimic the weathering process. Later, in 2005, Chen et. al [5] devised a new method of weathering called Y-ton tracing to extend to various materials 
and other types of weathering, such as the growth of moss, rust, dirt accumulation, and other sorts of weathering phenomenon.

\subsubsection{D Architectural Synthesis}

\section{Structures}

One of the first papers on the generation of procedural modeled buildings was by Wonka et. al [43] in 2003 in a paper titled Instant Architecture. They presented the notion of a "split grammar," a restricted, parametric grammar designed around the concept of shape. User input can guide the modeling process at any level, leading to very generic buildings or buildings adhering to user specifications very closely. Buildings with varying styles and designs can be generated in a highly automated way.

Later, more work was done on the procedural modeling of buildings by Muller et. al [26] through the derivation of a shape grammar named $C G A$ Shape that allows for creating building shells with incredibly high detail and visual quality, whereas the research by Wonka was more concerned with geometric detail on facades. The shape grammar allows modeling buildings using either approximate mass models or more complex shapes and roof surfaces.

Pottmann et al. [33] recently presented work in the architectural design of freeform shapes such as quadrilateral, pentagonal, and hexagonal meshes over curved surfaces. Given existing shapes and mesh qualities, they can find meaningful mesh and beam layout plans for the given input. Along those same lines, Smith et al. [38] presented a method for designing truss structures for buildings, bridges, roof supports, etc. Their method allows for the optimization of geometry and mass of structure, a non-trivial problem in the generation of complex man-made structures.

Bekins et al. [2] use photographs of buildings to design new buildings as well as model the original building from the photograph. They use a simple model recovered from a sparse image set and further refine the model in a procedural way that allows for the creation of new models, textured in the same way as the original model.

Hahn et. al [16] recently performed work in the real-time generation of persistent building interiors. Intended for use in interactive applications, it allows an application to produce interactive environments that are much larger than what can be locally stored in memory. This work is important because it adds another level of depth to urban modeling 
and synthesis, allowing even further complexity.

Havemann's [18] new Generative Modeling Language can define highly complex objects with a lot of regular, fine detail. Detail is defined using rules, making the language a highly efficient, compressed representation.

Recently, Merrell [25] described a method for synthesizing 3D models from examples. Given a small input model, his technique synthesizes new, larger and more complex models. This type of synthesis can be used to create models with various constraints, such as symmetry and change over time. His approach is similar to texture synthesis. He demonstrates his approach by synthesizing various collections of buildings.

Greuter et. al [14] presented a procedural approach to create geometrically varied buildings that are created on the fly. Building geometries are relatively simple and are extruded from generated floor plans. Caching and frustum filling manage which buildings are generated, and ensure interesting cities can be produced in real-time.

Cities without a population are ghost towns. Thomas et al. [40] populate virtual cities with animated cars and pedestrians.

\section{Surface Treatments}

In 2001, Dorsey et al. [21] described a method for texturing buildings with brick, tile, and stone. For such architectural elements, visual continuity across corners and seams is very important (see Figure 2.1). They use what they define as occupancy maps to "indicate which regions of a feature are already occupied by cells of its neighbors, and which regions remain to be textured."

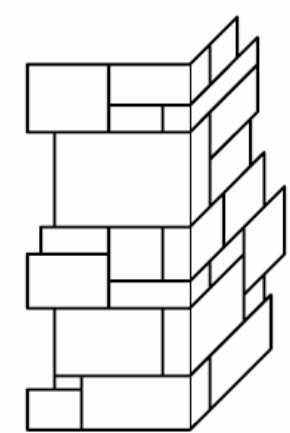

Correct

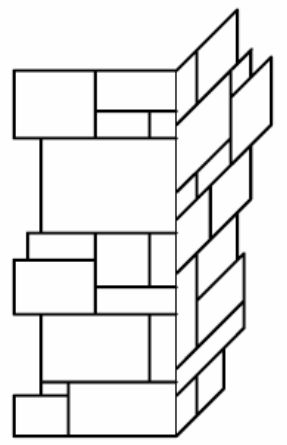

Incorrect

Figure 2.1: Example of building texture correctness from Dorsey et. al [21] 
More recently, Muller et al. [27] described methods for automatically generating 3D models of building facades from input imagery. By decomposing an input image of a facade, they can produce model generation rules encoded in CGA Shape form. Little user input is required, aside from indicating window depths.

\subsection{Generation of 2D Patterns}

\subsubsection{Texture Synthesis}

Texture synthesis is centered on the process of creating larger, sometimes more complex textures from smaller samples by analyzing the content of the provided texture. Our city generation problem is related because we are also interested in applying small-scale development patterns on a global scale. However, because we are modeling an entire, unique city with both local and global patterns, we cannot directly apply this sort of synthesis.

In 1985, Lewis [22] published one of the first papers looking at texture synthesis. He used a signal processing approach, enabling painting of texture with arbitrary spectra. Roughly 10 years later, Perlin and Vehlo [31] delved into the notion of multiresolution procedural textures to allow for texture refinement as the magnification of an image increases. Similarly, in our research we are interested in being able to represent our simulation results by vectorization so that at varying levels of magnification, we don't suffer from raster data becoming increasingly jagged.

More recently, Wei and Levoy [41] have extended Markov Random Field texture models to generate textures through deterministic searching. Their work can generate high quality, large textures synthesized from small texture samples. Ashikhmin's [1] work synthesizes naturally occurring textures, and allows the user to paint large-scale properties to guide synthesis. Similarly, in our city simulation the user can paint on a map to guide development.

Another way of synthesizing textures is image quilting, suggested by Efros and Freeman [11]. They stitch together small pieces of images and smooth the results. Their technique also allows for insertion of the texture into new images that may not be related to the synthesized image at all. More recently, Kopf et al. [19] extended the notion of texture synthesis to the 3D realm by synthesizing solid textures from 2D texture exemplars, and placing them on 3D model surfaces. Analysis of the surface to which the texture will be 
applied improves texture appearance further.

\subsubsection{Terrain Synthesis}

Terrain synthesis is procedural modeling of terrain. One of the first methods was described by Fournier et al. [13], which used stochastic processes to create natural irregular terrain. In particular, they used "fractional Brownian motion," developed by Mandelbrot. Later, Musgrave et. al [28] extended these fractal terrain models to add locally independent control permitting the modeling of erosion from water and thermal sources. More recently, Zhou et. al [45] extended texture synthesis techniques to create user-guided, synthesized terrain. Given digital elevation maps and a user sketch, synthesized terrain can be generated in the style of the user sketch in a way that mimics the patterns found in the elevation map data. Our simulation isn't concerned with modeling geographical features, but terrain synthesis can provide input on which our cities can develop.

\subsubsection{Urban Synthesis}

Our city simulation is a form of urban synthesis. Perhaps the first example of urban synthesis came from Stiny [39], who published an article in Environment \& Planning (B) that analyzed Palladio's system of architecture and derived a set of rules describing it. He organized these rules into a parametric shape grammar that generates ground plans, and used the grammar to generate the Villa Malcontenta, one of Palladio's villa plans. The grammar is derived from Palladio's I Quattro Libri dell'Architettura which sets out rules of classical architectural usage. Stiny's work laid some of the groundwork for procedural modeling. Many architectural synthesis techniques make use of grammars derived from Stiny's.

In 2001, Parish and Müller [29] presented research using L-systems to model cities. Their system takes images as input representing elevation, population density and road constraints, and generates highways and streets, subdivides land, then creates some building geometry. Figure 2.2 shows an example.

Harada et al. [17] described methods for designing architectural floor plans. Artists can interactively reposition rooms in a floor plan to see how that impacts automated repositioning of other rooms. 

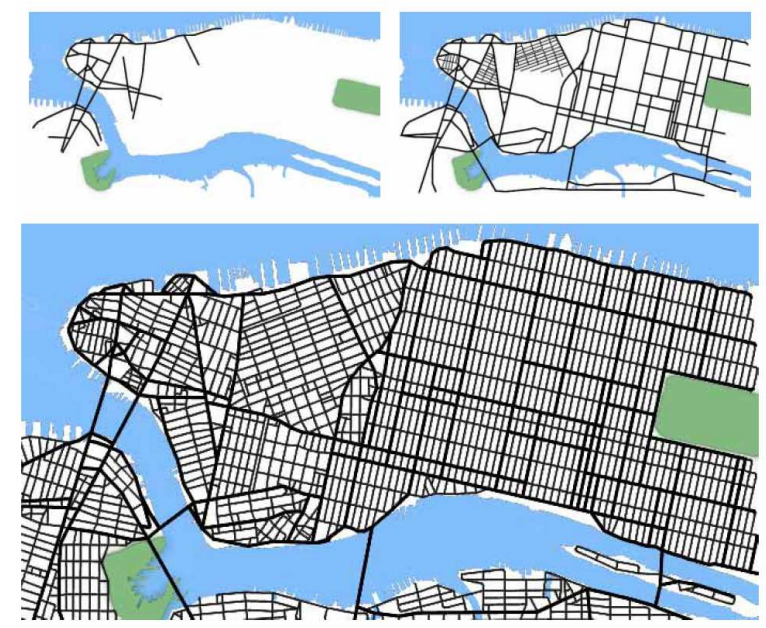

Figure 2.2: Example of road generation from Parish and Muller [29]

\subsection{Vectorizing 2D Patterns}

The subfield of computer graphics that may be most closely related to this research is image vectorization, which transforms image rasters into sets of continuous edges and curves. Our vectorization process differs from image vectorization by exploiting its noiseless input and knowledge of urban content. For a good summary of research on vectorization of line drawings, see [23].

Thinning or skeletonization of binary images is a fundamental image processing technique. Traditional thinning algorithms are computationally expensive and somewhat inaccurate. One of the first approaches to fixing these problems was published by Fan et. al [12]. They traverse a sparsely sampled version of the input image, creating an orthogonally zigzagging set of connected bars, which are used to generate a skeleton. Later, Liu and Dori [8] extended this idea to create the Sparse Pixel Vectorization (SPV) algorithm, which starts from meaningful medial axis points in the image, generalizing the image traversal and improving junction recovery, and avoiding the dips that often occur at skeletal joints.

In 2001, Zou and Yan [46] used constrained Delaunay triangulation (CDT) to generate skeletons. First, the CDT is created for the passed input. Then, this triangulation is used to obtain a skeleton by analyzing the internal triangles of the shape. Afterwards, artifact removal occurs to remove periphery skeleton branches that meet specific criteria. Intersection artifacts are also removed by the merging of intersection triangles into a region 
where a new intersection point is created.

These techniques for the vectorization of line drawings provide groundwork for further, more complex applications of the vectorization techniques. Chiang, Knoblock, and Chen [6] devised a method of taking raster road maps and matching them to their vector or satellite data equivalents by extracting information about road intersections and performing various image processing techniques to simplify complex raster data. With morphological operators such as dilation, erosion, and thinning, they can clean up extracted road information and create fingerprints of local areas by extracting locations of road intersections from the cleaned data. They match this fingerprint with known vector data, allowing for the two data sources to be paired.

In 2006, Mena [24] published work on a new method to vectorize segmented road networks by analysis of high-resolution imagery. First, the image is denoised. Next, parallel edges of roads are smoothed, a skeleton is extracted, and the graph and actual road network are created. They also correct intersections in the road network, which often is distorted during vectorization. Figure 2.3 shows an example.
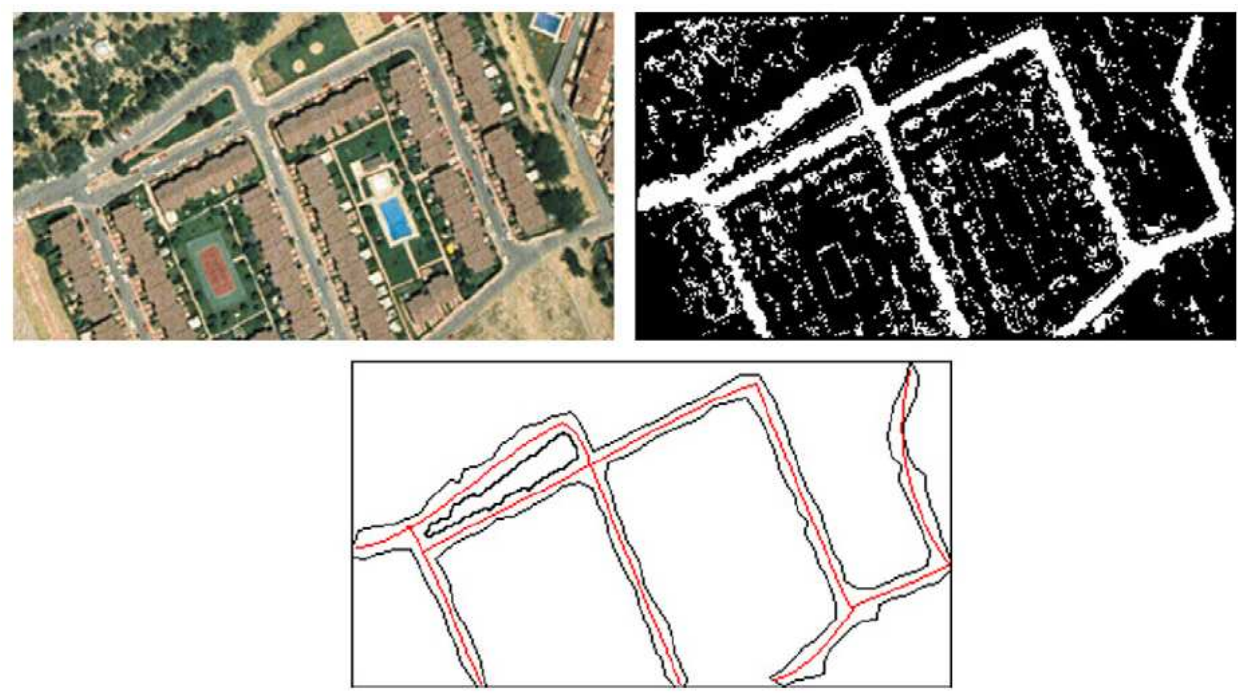

Figure 2.3: Extraction of segmented road network by Mena [24]

Other papers such as Pless and Jurgens [32], Chen et al. [4], and Gyftakis et al. [15] are also concerned with the extraction of road information from photography. All of these methods make use of much higher resolution input than we do. 


\section{Chapter 3}

\section{Framework}

\subsection{Initial Data Preprocessing}

\subsubsection{Input Data Loading}

We have two basic forms of input data from the city simulation. The first is the gridded land use data. Each patch (grid element or cell) in the data represents one of the following types: water; secondary or primary road; residential, commercial, residential, park or reserved uses; or unused land. The second form of data is comma-delimited list information regarding parcels (properties). Each parcel contains information about its developed density, type of land use, and member patches. The initial data can be seen rendered in Figure 3.1.

\subsubsection{Raw Block Generation}

After loading the land use data into a simple array, we create blocks. There are two different types of blocks: internal blocks are completely surrounded by existing roads, while external blocks are not. External blocks are found on the edge of the city, where development continues (or has been constrained by the end of the simulated world). Internal blocks are made up of patches that are not part of roads, bodies of water, or reserved parcels. We construct them using a "flood fill": beginning with a seed patch, we visit all patches adjacent to the partially completed block and add them to the block if their type is appropriate. (Note that two or more blocks may be surrounded by the same roads when water or reserved land is also surrounded by these roads). We compute external 


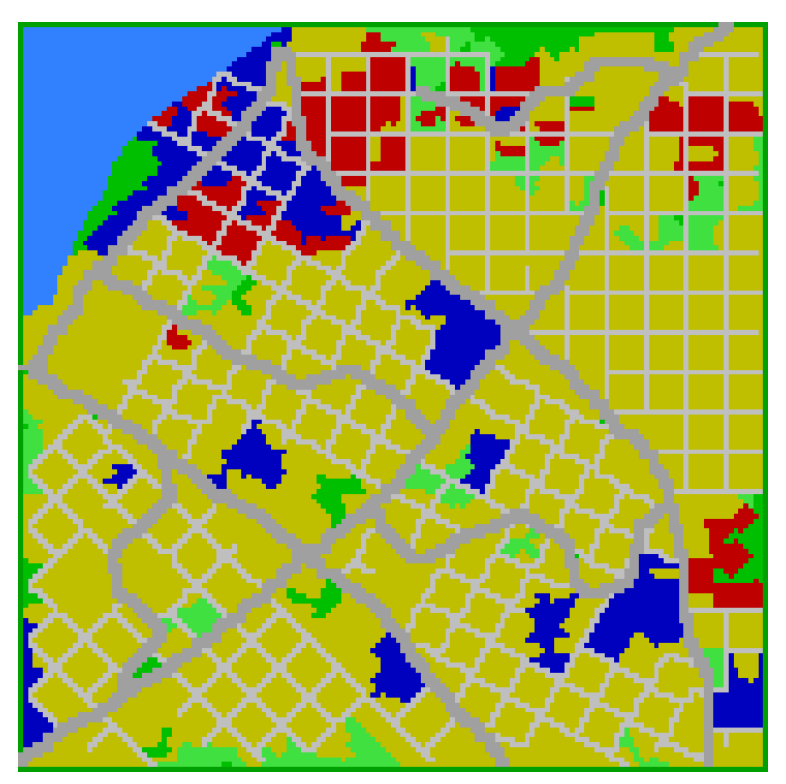

Figure 3.1: View of initial data from simulation

blocks similarly, but also exclude undeveloped land.

\subsubsection{Extraneous Intersection Patch Cleaning}

Because the simulation occurs in a gridded environment, there may be primarysecondary road intersections that have ambiguous direction. These ambiguities result when there are redundant connections between secondary and primary roads. We eliminate these redundancies to fix most of these ambiguities. From each secondary road patch adjacent to a primary road, we visit all four-connected secondary patches, halting at any disconnected patches. In this case a patch is disconnected if it is neither four- nor eight-connected to a primary road patch (a grid element $(x, y)$ is eight-connected to all of its four-connected neighbors, as well as the elements at locations $(x+1, y+1),(x+1, y-1),(x-1, y+1)$ and $(x-1, y-1))$. If we do not reach any disconnected patches, then all visited patches are directly adjacent to primary road and may be deleted. If we do reach disconnected patches, then for each disconnect we save only one path back to the primary road. The patches in this path are in the shortest path between the disconnected patch and the closest patch that is four-connected to primary road. We remove all visited patches not in one of these shortest paths and give them a type that indicates they were once road but are no longer. 
This can be seen in the green squares in Figure 3.2.

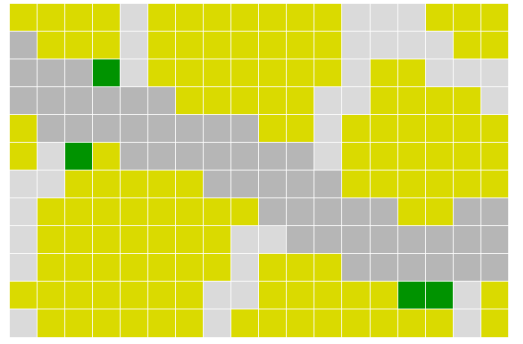

Figure 3.2: Extraneous road patch removal

\subsubsection{Road Graph Generation}

We next build an undirected graph from the road patches found in the input data. We begin by locating the graph nodes. We iterate over all patches in the data set, treating primary and secondary road patches differently, because primary and secondary roads have different widths (secondary roads are one patch wide, primary width are two patches wide). We locate the graph node for secondary road patches at the patch center. Primary road patches receive a graph node only if their east, southeast, and south neighbor patches are also primary roads, effectively creating primary road graph nodes only when the corresponding patch is at the center of the wider primary road. Primary graph nodes are located at the center of the corresponding patch's southeast neighbor.

We now revisit the patches in the simulation data to connect the graph nodes. A road patch's node is linked to the node corresponding to any four-connected road patch. Note that many primary road patches will not have a corresponding graph node, and will therefore not become part of the road graph. At this stage in the process, because graph nodes are located at patch centers, links are oriented in one of the four cardinal directions. Figure 3.3 shows an example.

To eliminate very short graph cycles, we iterate through all graph nodes, attempting to follow links in the south, east, north and west directions. If we are able to follow all of these links, we know there is a short cycle in the graph, representing four patches organized into a $2 \times 2$ square. To eliminate it, we find the patch four-connected to two patches that aren't road, remove it from the graph, and mark the patch as removed road. This stage can be seen in Figure 3.4. 


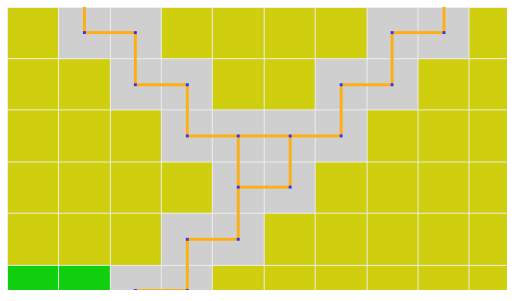

Figure 3.3: Graph connectivity before cycle elimination

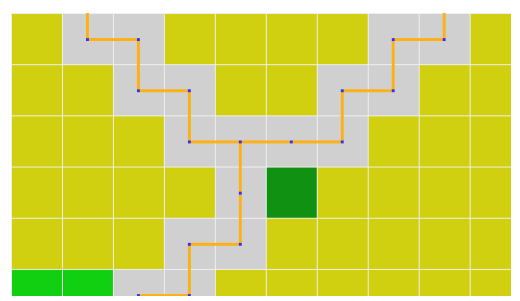

Figure 3.4: Graph connectivity after cycle elimination

We now examine all graph nodes to identify intersections, which are defined as nodes having more than two links, or linked only to other intersections. Having found these points, we then replace clusters of adjacent intersections with one simplified intersection, unless the intersection node would only be connected to two other nodes (See Figure 3.5). We find the location for simplified intersections by averaging the location of the unsimplified adjacent intersections.

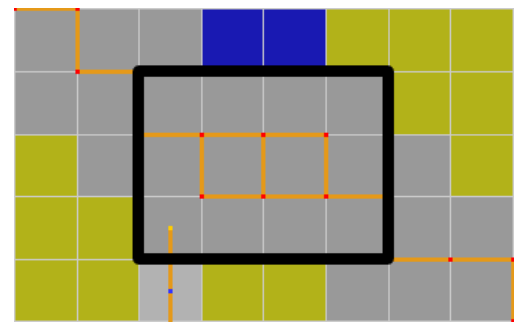

Figure 3.5: Cluster of loops that don't represent an intersection

We now enter a logistical and organizational phase. Until this point, the graph was represented using a (partially filled) four-connected lattice corresponding to the simulation grid, enabling us to use techniques dependent on cardinal direction or grid adjacency. We 
now discard this representation in favor of the more traditional, unconstrained and efficient representation that contains only those links a node actually possesses. We also look for linked graph nodes that are more than two patch widths away from each other, eliminating such long links by inserting nodes until all resulting links are shorter than two patch widths. This ensures that all nodes have roughly the same weight in the filtering process described below. Finally, we place every node's links into a clockwise ordering from due north. This step supports inferences about turning direction.

Because roads form the perimeters for most blocks, it is now advantageous to construct external perimeters for any external blocks found earlier. Such perimeters will have undeveloped land on one side, and developed land on the other. We therefore gather a connected list of all patch vertices (or corners) that bound an undeveloped patch. Two vertices in this list are connected only if the edge between them is not developed on both sides. To build perimeters, we first search for a vertex in our list that only has one point connected to it (such points are located where the perimeter meets the road). Starting from this point, we walk the connectivity in our list, inserting visited vertices into our perimeter list and removing them from our bounding list. When connectivity ceases and the other side of the perimeter is reached, we check the bounding list, and if it is not empty, begin working on the next perimeter. We convert the resulting perimeter lines to a series of nodes and links connected to our road network. Finally, we are left with a network ready for manipulation (See Figure 3.6).

\subsubsection{Parcel Data Loading}

As noted earlier, our urban simulation outputs both map and parcel (patch grouping) data. Parcel data includes development type (e.g. commercial, residential), patch count, population, and a list of patches in the parcel. To associate this data with the simulated blocks we generated directly from the map data, we find the simulated block that contains the first patch in each parcel. 


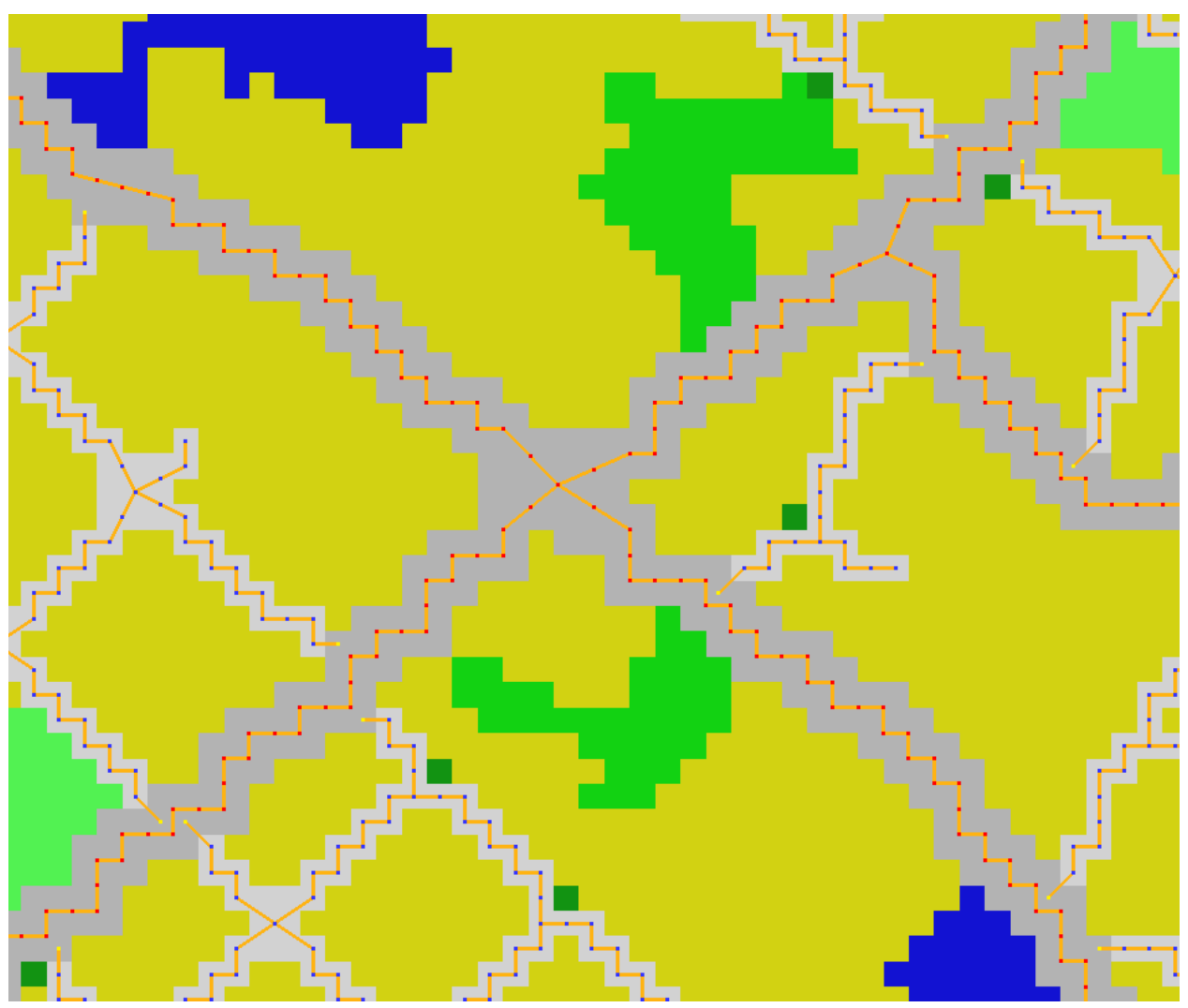

Figure 3.6: Data ready for manipulation and processing

\subsection{Road Correction}

\subsubsection{Intersection Straightening}

We now begin a number of steps designed to reposition roads to improve appearance. We start by straightening intersections, which due to the input simulation grid are only coarsely aligned. At each intersection, for each pair of incoming roads, we evaluate their fit to a line. If that fit is reasonably good, we snap road nodes near the intersection to the fitted line. We use a linear regression on nodes in the pair that are at most five patch widths from the intersection. We define "reasonably good" to be a residual norm of 1.2 or less (an experimentally determined value that is slightly over one patch width). For an example of intersection straightening, see Figure 3.7. 

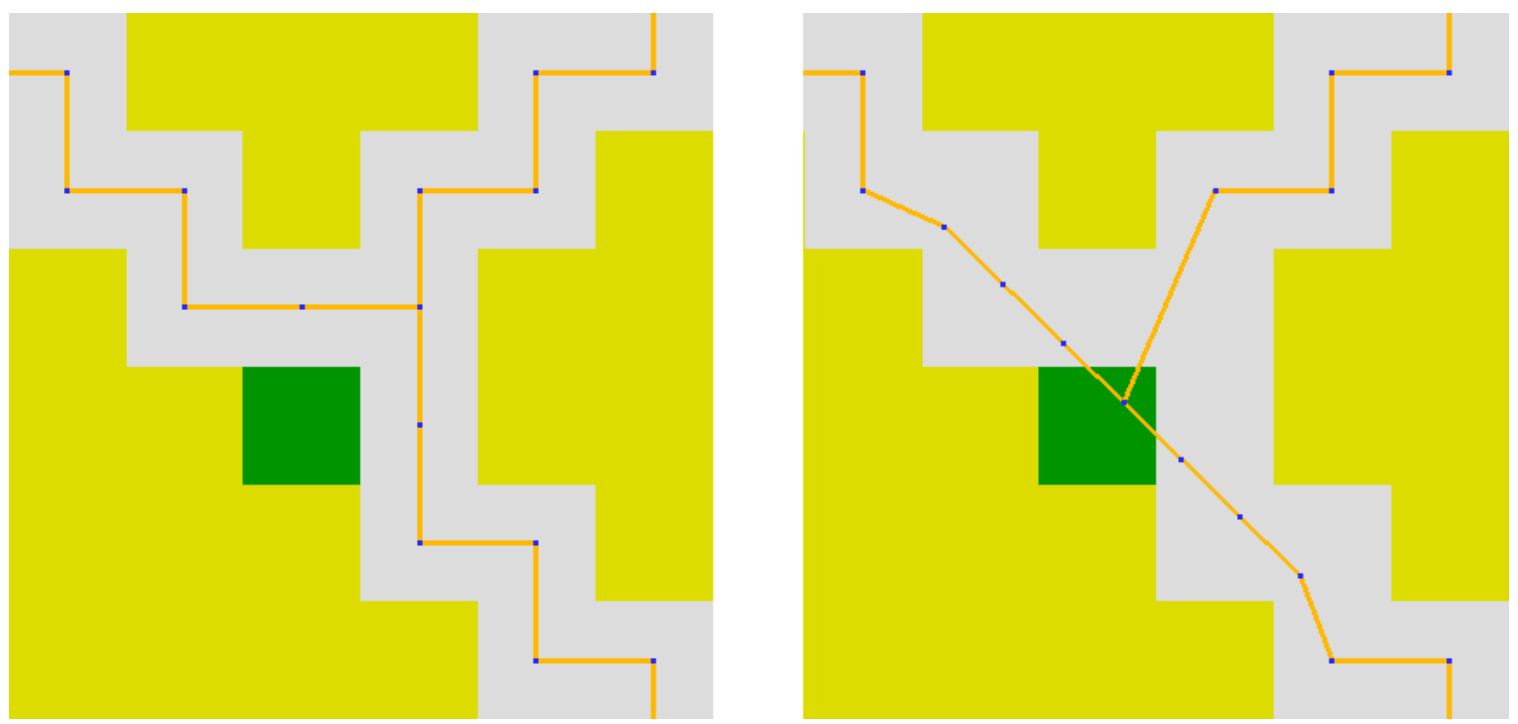

Figure 3.7: Intersection straightening (Left: before, Right: after)

\subsubsection{Road Smoothing}

To smooth roads, we average the locations of connected road nodes. We do not initially smooth nodes at dead ends, intersections or primary/secondary road connections. Averaging only includes nodes of the same road type, and only occurs when there are at least two linked nodes. Having repositioned roads outside of intersections, we straighten intersections again. We calculate the dot product between all pairs of incoming roads, and when the angle between the pair approaches 180 degrees, we simply place the intersection on the line between the two closest nodes on the incoming road pair. An example of how smoothing operates on the network can be seen in Figure 3.8.

\subsubsection{Primary and Secondary Connection}

Until this point, primary and secondary road graphs have been completely disjoint. This is a natural consequence of their separate land use types in simulation output. Having connected, cleaned and smoothed both graphs separately, we merge them into one graph. Nodes that must be merged correspond to secondary road patches that are four-connected to a primary patch. We merge these nodes by linking them with the closest node in the primary network. After all such merges, we simplify any resulting intersection clusters, as 


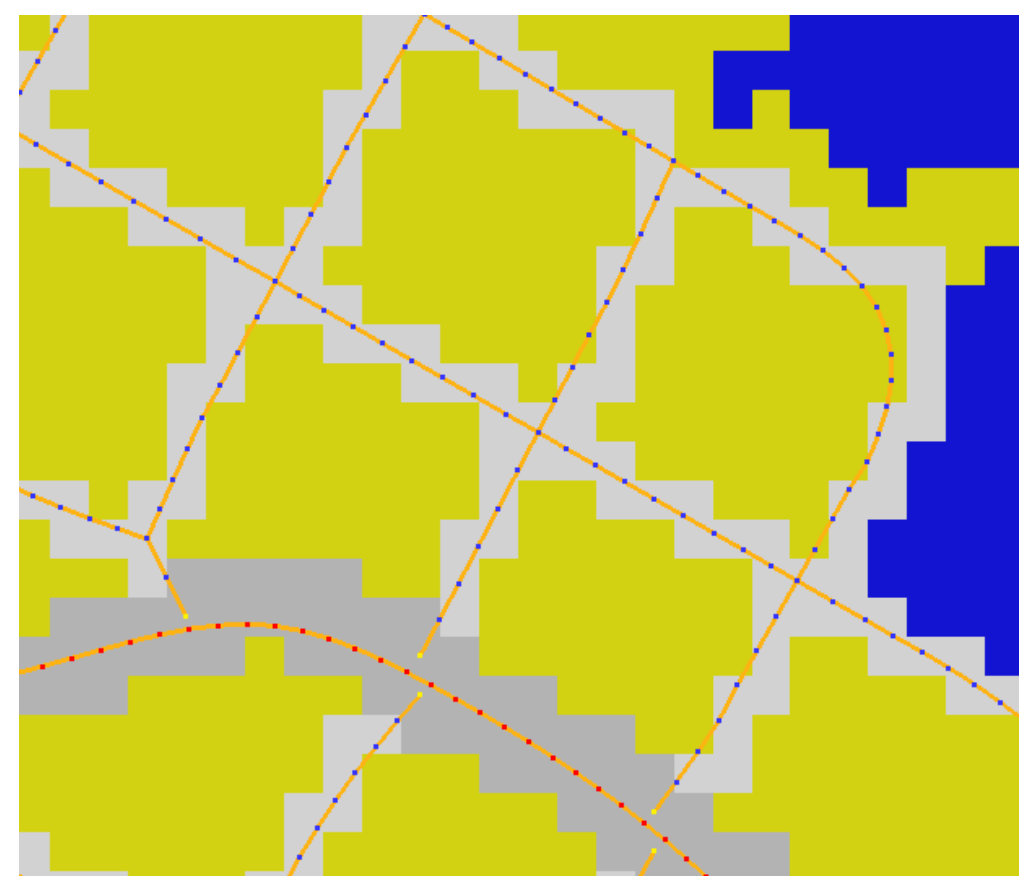

Figure 3.8: Road smoothing and intersection straightening

described above. The end result of this pass can be seen in Figure 3.9.

We use a similar process to connect external block perimeters to the road graph, producing a complete partitioning of the map into blocks. In this case, perimeter ends are connected to any road node not already connected to a perimeter.

\subsubsection{Cycle Removal}

Simulation, cleaning and smoothing can sometimes produce short road segments enclosing undeveloped land that is awkward to develop. Figure 3.10 shows such segments. We eliminate these by locating cycles in the road graph of 15 nodes or less, and removing one segment of road.

\subsubsection{Intersection Simplification}

We now simplify overly complex intersections to avoid problems when we eventually add road geometry such as curbs. We remove any dead ends connected directly to the intersection and angled within 90 degrees of another road. We also remove any nodes that 


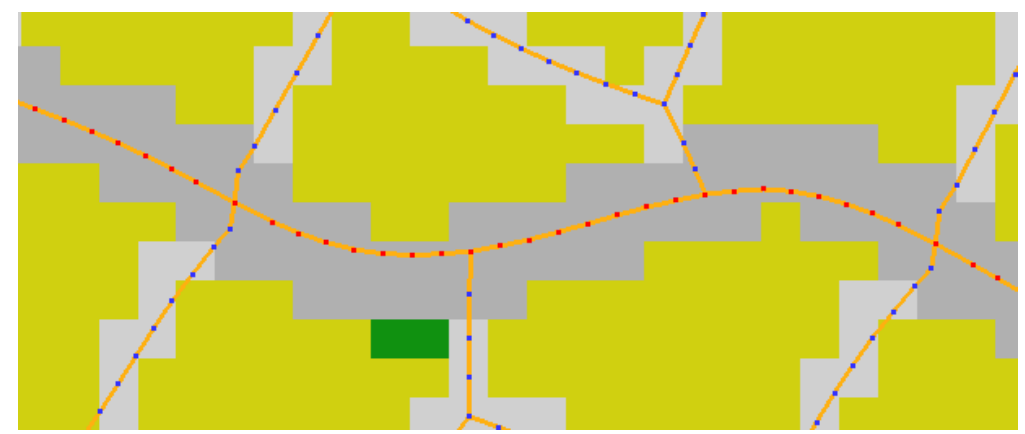

Figure 3.9: After connecting secondary and primary
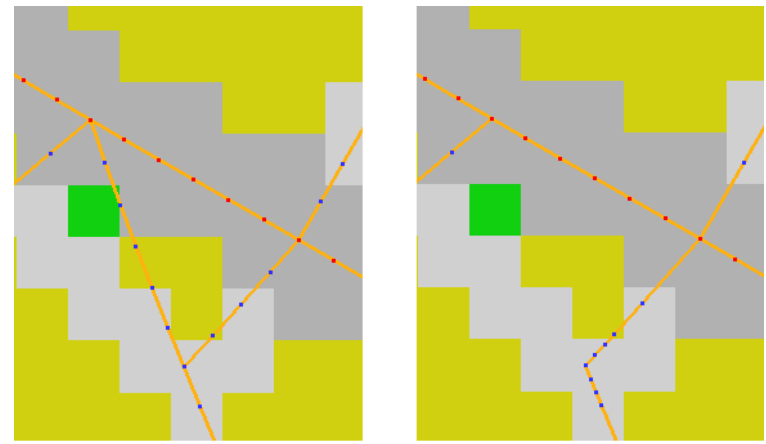

Figure 3.10: Short cycle to be removed (Left: before, Right: after)

are extremely close to the intersection. Primary nodes are removed if they are within one patch width, secondary nodes if they are within $1 / 2$ patch width.

\subsubsection{Curve Smoothing}

We now smooth the road graph further (eight smoothing passes seems to work well for most simulation output). To avoid over-smoothing of high frequency features, we add nodes where curvature is high. If the angle defined by three consecutive nodes outside an intersection is less than 90 degrees, we add three nodes on each side of the central node. If the angle is less than 135 degrees, we add two nodes per side, and if it is less than 160 degrees, we add one per side. Figure 3.11 shows an example. 

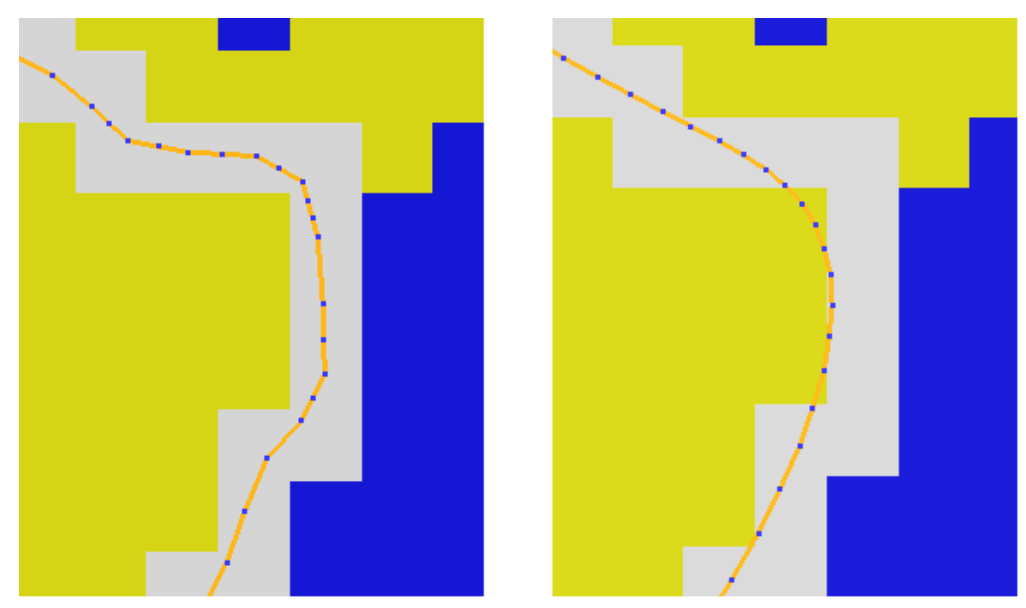

Figure 3.11: Increased point count in curves preserves features

\subsection{Road Geometry Creation}

We now add curbs, pavement and culs-de-sac to the road graph. (We leave external block perimeters unchanged). Primary curbs are one patch width away from the graph nodes, while secondary curbs are half a patch width away. Curbs are oriented parallel to the graph links in their segments, and pavement is a triangle strip using curb vertices as its vertices. At dead ends on secondary road segments, we add octagonal culs-de-sac.

Road segments that terminate at intersections require more complex processing that ensures that their pavement does not overlap. Processing proceeds by considering curbs attached to each adjacent pair of road segments, when an intersection's road segments are ordered using a consistent clockwise winding. The result of processing each pair is a curb corner. Each road segment in the current pair has two parallel curbs. Each such curb intersects both of the parallel curbs from the other road segment, forming four candidate corners. We find the correct corner by inserting all candidates into the intersection's winding order, and choosing the corner between the current road segment pair. Note that when the segments in a pair are parallel or nearly so, no corner is actually required, and in fact the floating-point calculation of any curb intersection is unreliable. In such cases we simply add an edge between the ending vertices of two of the road segment curbs. We identify the two curbs to connect in this way by inserting all four curbs for the pair into the intersection's winding order. The two curbs to connect will both be located between the current road 
segment pair.

With these corners we can trim our road segment triangle strips, and construct a convex intersection polygon. Road segment pavement terminates with an edge between the corners of each of its curbs. We take special care during trimming to maintain the integrity of the triangle strips representing roads. Intersection pavement is represented by a convex polygon constructed from the corner vertices, in clockwise intersection order. Figure 3.12 shows road geometry.

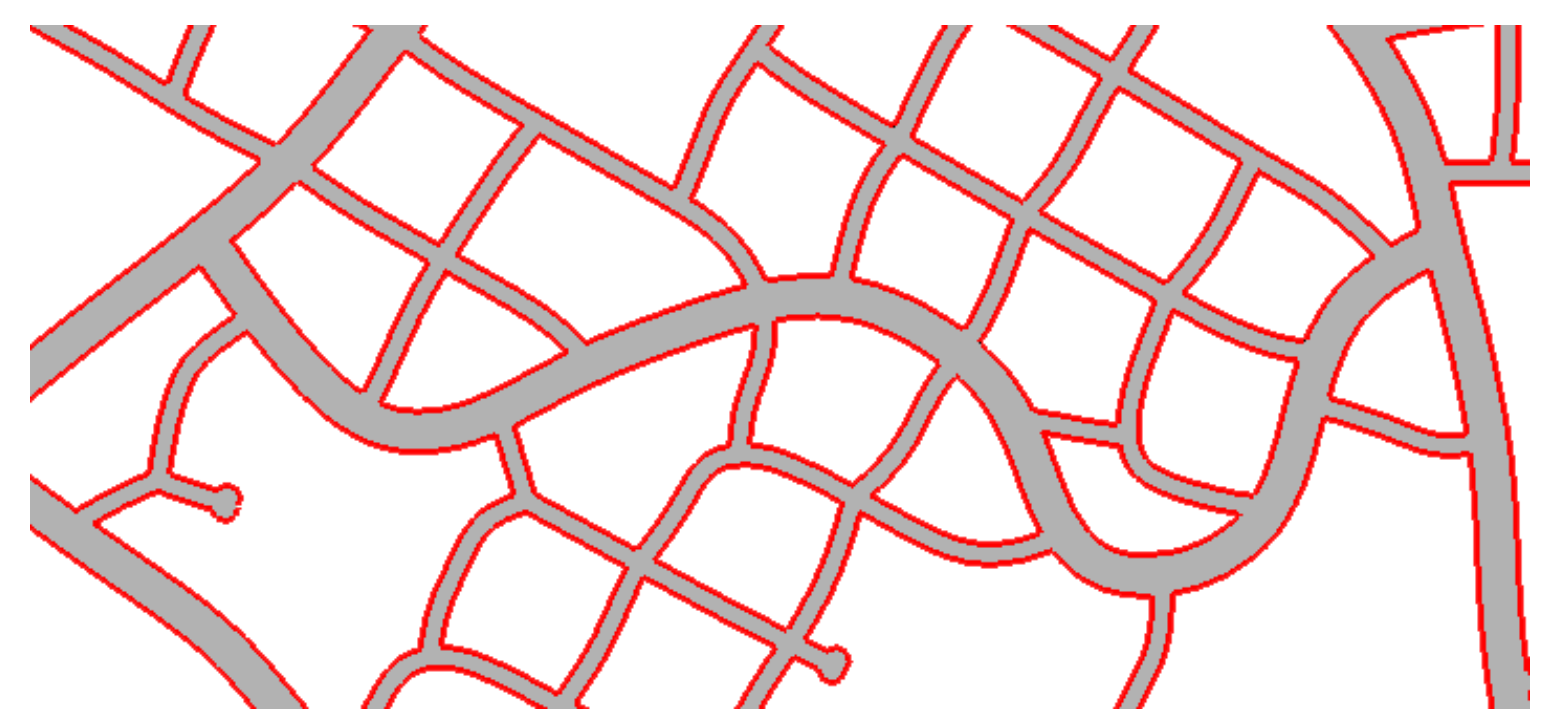

Figure 3.12: Road geometry with road edges highlighted

\subsection{Vectorized Block Development}

We now create vectorized blocks to match the blocks we extracted from the input simulation results. Vectorized blocks are based on road curbs and the original external block boundary lines we built earlier.

To find all of our internal blocks, we follow road edges or links in a consistent direction (e.g. turning right for a clockwise order). Each time we reach our starting point, we enclose a new block. One resulting "block" is in fact the entire outer boundary of our road network. We save this perimeter for later use, but discard it from our current list of vectorized blocks. While we build each block, we create an oriented bounding box for it using principal component analysis and calculate its diagonal. 
Blocks containing water should not include that water. We therefore split these blocks using water boundaries. We identify simulation blocks containing water, identify the corresponding vectorized block, and insert a smoothed version of the water boundary in the simulated block into the vectorized block. See Figure 3.13 for an example of this water line detection.

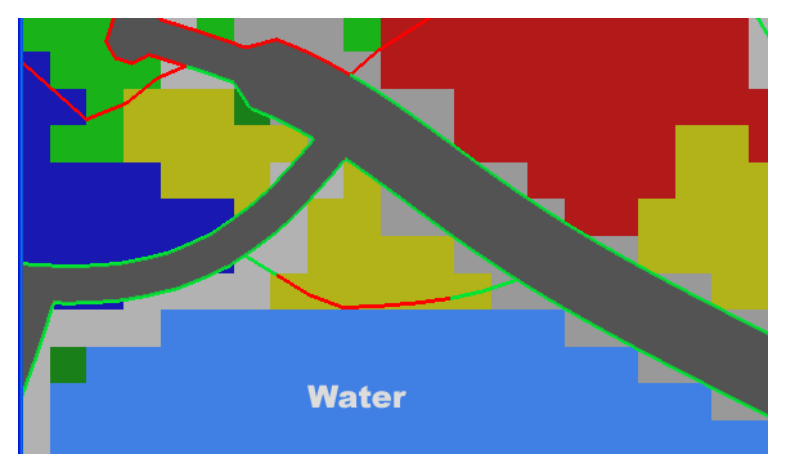

Figure 3.13: Example of block generated from water line

We vectorize external blocks using the external block boundaries generated earlier, and the external road perimeter that we saved during the creation of internal city blocks. We begin by reconnecting the two endpoints of each external block boundary back to the external road perimeter. We then transform each such boundary into a completely closed block boundary by exploiting the perimeter's clockwise ordering (recall that like internal blocks, the perimeter lists road nodes in clockwise order). Vectorized external block boundaries begin at the lower-numbered (in the clockwise perimeter order) end of the saved portion of the external block boundary, connect back to the road perimeter, and are then closed by following the perimeter in counter-clockwise order. If an external block has two or more saved external boundary lengths (meaning there is undeveloped land bounded by the road perimeter and the external block), then it is followed rather than the road perimeter. The external block is completely enclosed when we reach the starting point of our traversal.

Road smoothing at particularly sharp turns can leave some external blocks inside the smoothed road perimeter. We identify such blocks by finding those with boundary lines with both end points inside the road perimeter and with more than $50 \%$ of nodes inside perimeter, and effectively delete the corresponding blocks by removing these boundary lines 
before the corresponding external blocks are vectorized. (Future versions of this vectorizer should probably use a more robust identification algorithm that deletes external blocks if there is an intersection of any kind between the boundary and the perimeter). We show an external block in Figure 3.14.

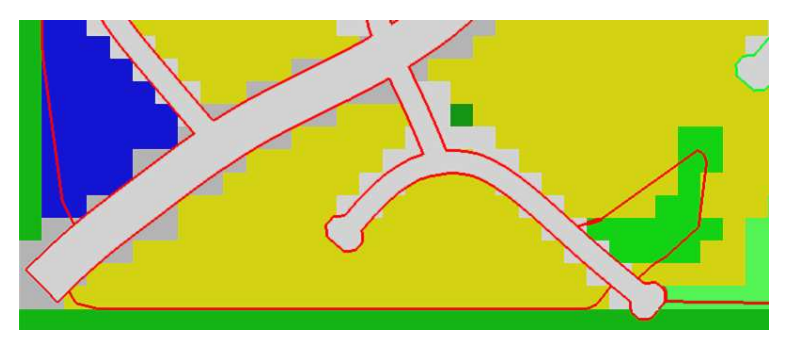

Figure 3.14: Example of block generated from external boundary lines

\subsubsection{Matching of Simulation Blocks to Generated Blocks}

To associate all newly generated vectorized blocks with the simulation blocks, we calculate the center of each simulation block (unless the block isn't already matched to a vectorized block, which might occur when resolving water boundaries). If the center is inside the simulation block, we look for the vectorized block that contains the center and associate it with the simulation block. If the center isn't actually in the block (e.g. the block is highly concave), we instead use the parcel center that is closest to the block center, ensuring that that the center is actually inside the block. If there are any simulation blocks that aren't associated with generated vectorized block, we assign them to the vectorized block that has the center closest to the center of the simulation block. We do not often make use of this failsafe.

\subsubsection{Generation of Polygonal Spines}

When city blocks are more rectangular than square, parcel boundaries often form a "spine" aligned with the long axis of the block. These spines ensure efficient and profitable allocation of property. We define such "oblong" blocks as those having an aspect ratio less than 1:2. We compute the aspect ratio using the bounding box generated previously. In addition, any block containing a cul-de-sac receives a spine: such dead ends are only inserted into blocks when interior parcels need access to the road network, and a spine 
ensures that these interior parcels will be created. We do not create spines on any exterior block, because they would make the parcels on the exterior side of the parcel inaccessible.

We build spines by making a binary image approximation of the block, performing constrained Zhang/Suen image thinning [44], and then converting the image back into a vectorized representation. (Other thinning methods would generate slightly improved spines, but they are much more complex and less efficient). To convert the block into a binary image, we rasterize the block's axis-aligned bounding box using eight pixels per patch. If a pixel is inside our block's polygon, it is set to black, otherwise it is set to white. After thinning, we re-vectorize the result by adding edges between all four-connected spine pixels. In any remaining points that have one or fewer edges, we add edges between any eight-connected neighbors. We then smooth the resulting vectorized spine by averaging vertices with their neighbors, prune any short branches off the primary spine that are less than two edges in length, and trim any spine ends that are within one patch width of a block edge. Finally, we make the spine look more like a manmade, piecewise-linear polyline using simplification. This process traverses the spine, summing edge-to-edge turning angles at each vertex. When this sum exceeds 20 degrees (an empirically determined threshold), we use an edge to replace all the vertices between the vertex at which summing began, and the vertex at which the threshold was exceeded. To make parcel simplification easier (see below), we ensure that spine vertices containing more than two edges are not directly connected by a single edge. Figure 3.15 shows examples of generated spines.

\subsubsection{Subdivision of Blocks}

Urban blocks are divided into separately owned parcels. If a block has a spine, we begin by first placing parcel boundaries at all spine ends. If the block has no spine, or the number of spine-based parcels is still less than the number of parcels in the corresponding simulated block, we subdivide further. To match the vectorized subdivision as closely as possible to the simulation's subdivision, we generate many (currently 50) randomized subdivisions, and pick the subdivision that matches the simulation subdivision most closely. We use this generate-and-test paradigm because the simulation grid's resolution makes its parcel boundaries extremely coarse, and only loosely defines the target subdivision. 

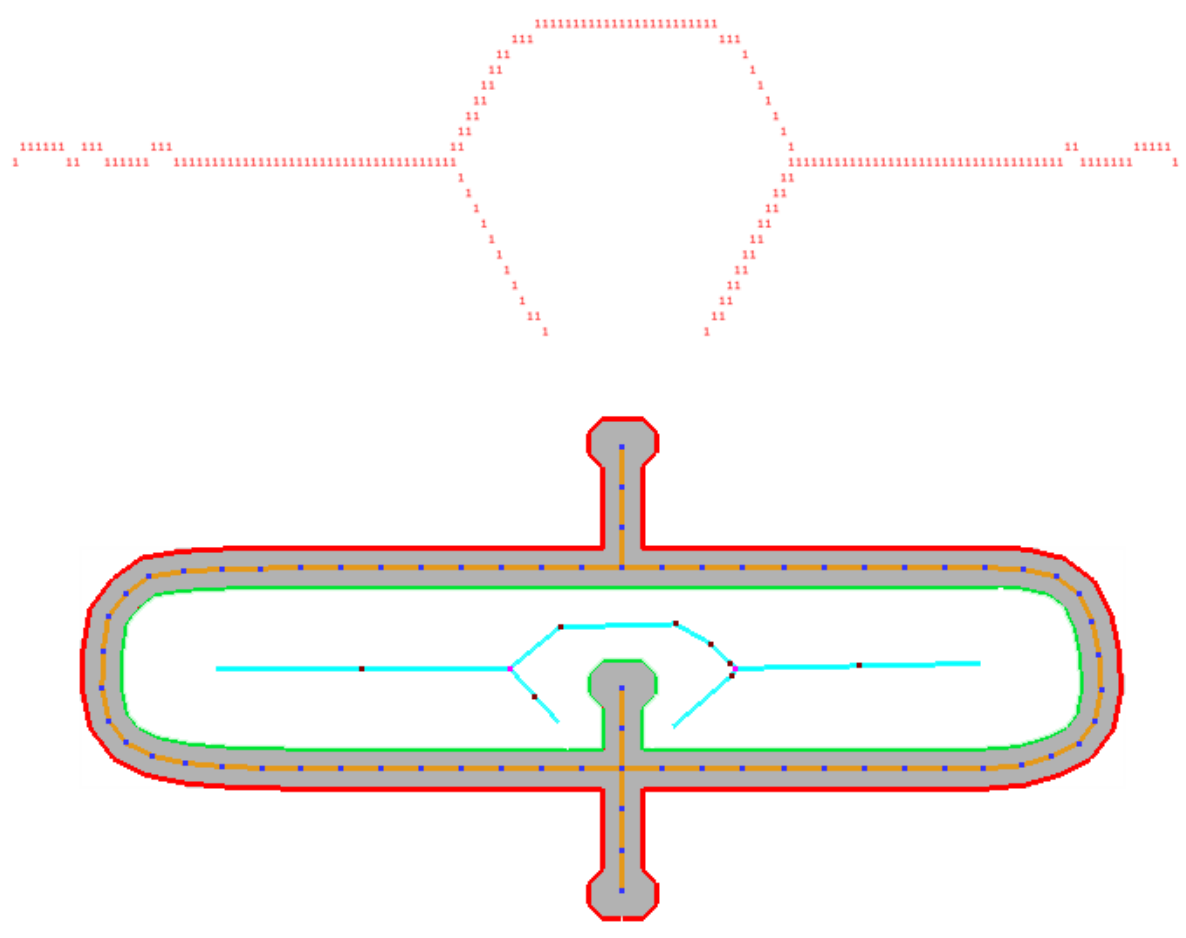

Figure 3.15: Spine generation: Top, binary. Bottom, polygonal.

\section{Spine-Based Subdivision}

If the number of parcels in the simulated block is greater than the number of spine ends in the vectorized block, then spine-based parceling will create too many parcels. We therefore discard the spine completely, and do not create spine-based parcels. Otherwise, we visit each spine end and create a parcel-bounding edge between the end vertex and the closest point on the vectorized block. Figure 3.16 shows the result of spine-based subdivision.

\section{Regular Subdivision}

We begin regular (non-spine-based) subdivision by making a copy of the block. We repeatedly split the largest remaining parcel in a constrained, random fashion, continuing until we produce the number of parcels in the simulated block. Each split creates two new parcels with a new parcel edge that splits and is perpendicular to a road-facing edge. We choose the road-facing edge randomly and place the new parcel edge at a random location biased toward the center of the road-facing edge using a normal distribution. To avoid 


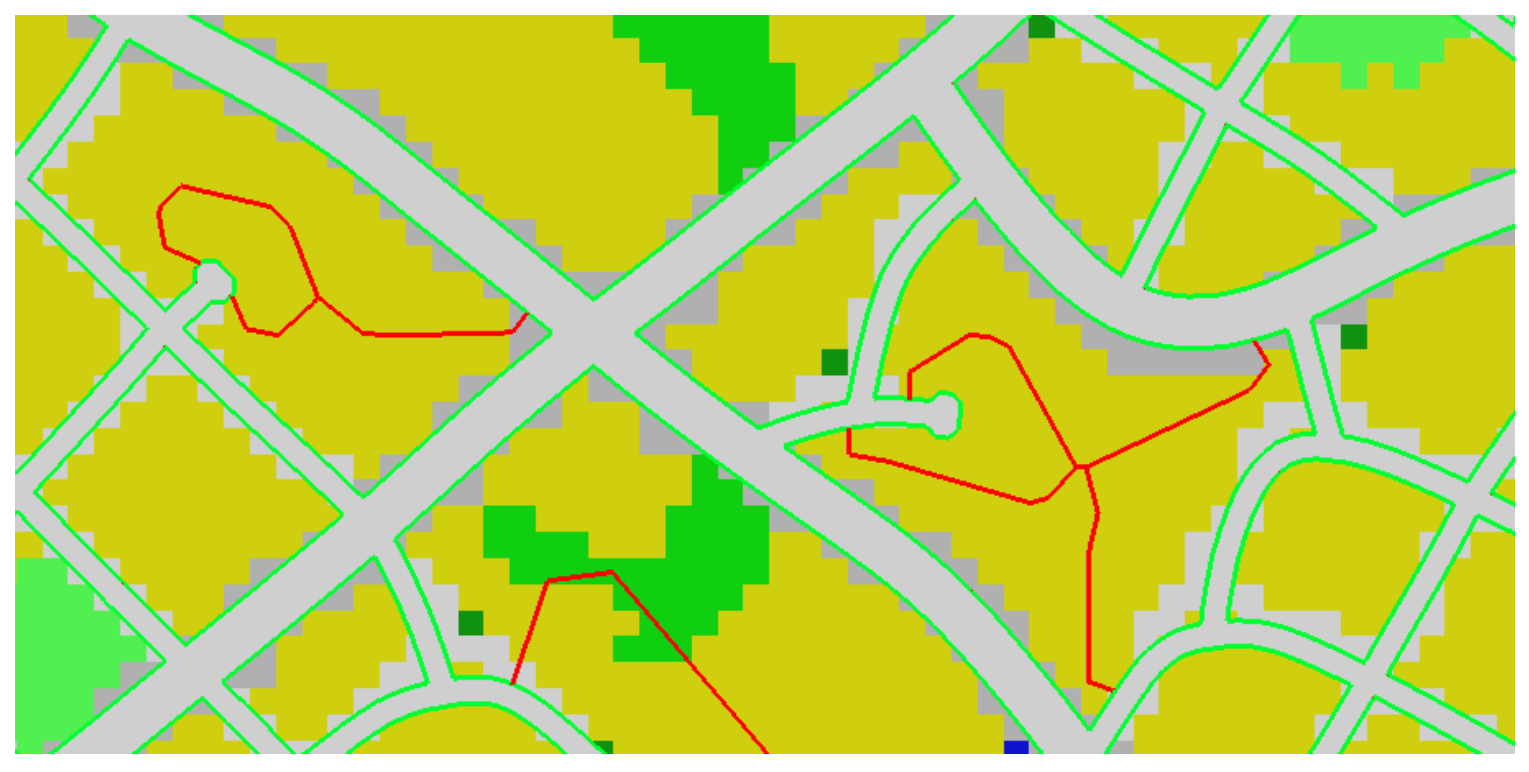

Figure 3.16: Example of generated spines for blocks

inappropriate splits caused by local perturbations in the block boundary (which contains edges with length of roughly one patch width or less), we simplify the boundary of the block's copy, and perform subdivision on that simplified block. We then transfer each split to the original vectorized block, where it may not be perpendicular to the road-facing edge it intersects. We evaluate this split against the following constraints:

1. Minimum parcel size: each new parcel must have at least $75 \%$ of the area of the minimum parcel size in the simulated block.

2. Road access: each parcel must have a road facing edge that is at least 0.5 patch widths in length.

3. Limited rectangularity: if the original parcel had an aspect ratio greater than 0.25 (not too rectangular), the two generated parcels must have an aspect ratio that is at least as large (not more rectangular). If the original parcel has an aspect ratio less than 0.25 (too rectangular), both of the two generated parcels must increase the aspect ratio (be less rectangular).

4. Limited variation in residential parcel size: If residential land use fills $70 \%$ of the block, parcels must be roughly equal in size. Neither parcel may have less than $30 \%$ 
of the original parcel's area.

If the new parcels meet these constraints, we allow the split and attempt the next. If we cannot generate an acceptable split of the largest parcel after multiple attempts, we attempt to split a random parcel.

Because the simulation does not explicitly output undeveloped block parcels, we will have to subdivide some vectorized blocks into more parcels than the simulation explicitly indicates, in order to create these undeveloped "negative" parcels. If the proportion of undeveloped land in a block is more than $25 \%$, we mark it for additional subdivision and parceling. We could determine the number of additional parcels to create by counting the number of disjoint regions of undeveloped land in the simulated block, however many of these regions are too small to bother recreating. Our current heuristic is to double the number of undeveloped parcels already created. The added parcels provide enough additional granularity for our vectorizer to maintain the overall proportion of developed to undeveloped land (see land use assignment below).

\section{Use Assignment}

We assign uses to newly subdivided blocks by attempting to reproduce the distribution of uses in the corresponding simulated block. In a not uncommon trivial case, all parcels in the simulated block have the same land use. If such blocks are also at least $75 \%$ developed, we simply assign all parcels in the vectorized block the same use. Otherwise, we assign land use stochastically, using a distribution based on differences between the vectorized block and the corresponding simulation block. In particular, the distribution is shaped by distance between parcel centers, proportions of use in the blocks and differences between parcel sizes.

For a certain vectorized parcel, the distance from its center to the center of the nearest simulated parcel center with use $i$ is $d_{i}$. The distance-based probability that the vectorized parcel will have use $i$ is $p d_{i}$ :

$$
\begin{gathered}
d_{i}=\text { distance to use i } \\
p d_{i}=\frac{d_{i}^{-1}}{\sum_{i} d_{i}^{-1}}
\end{gathered}
$$


The proportion of land dedicated to use $i$ in the simulated block is $u s_{i}$. If the proportion of undeveloped land is less than 0.25 , then $u s_{i}$ is

$$
u s_{i}=\frac{\sum \text { area of parcels with use } i \text { in sim block }}{\text { area of developed land in sim block }}
$$

Otherwise, $u s_{i}$ is:

$$
u s_{i}=\frac{\sum \text { area of parcels with use } i \text { in sim block }}{\text { area of land in sim block }}
$$

Similarly, $u_{i}$ is the proportion of land in the vectorized block dedicated to use $i$ :

$$
u_{i}=\frac{\sum \text { area of parcels with assigned use } i \text { in vectorized block }}{\text { area of vectorized block }}
$$

With these definitions, we can define the unnormalized, proportion-based probability that the vectorized parcel will have use $i$ as $p u u_{i}$ :

$$
p u u_{i}=\max \left(0, u s_{i}-u_{i}\right)
$$

The analogous normalized probability $p u_{i}$ is:

$$
p u_{i}=\frac{p u u_{i}}{\sum_{i} p u u_{i}}
$$

To add a size-based component to our probability distribution, we define $a s_{i}$ as the average size of parcels with use $i$ in the simulated block. We define $d s_{i}$ as the standard deviation of the size of parcels with use $i$ in the simulated block. We further define $a$ as the area of the current parcel in the vectorized block. The size-based probability that the vectorized block has use $i$ is $p s_{i}$ :

$$
p s_{i}=\frac{\operatorname{normal}\left(a ; a s_{i}, d s_{i}\right)}{\sum_{i} \operatorname{normal}\left(a ; a s_{i}, d s_{i}\right)}
$$

where $\operatorname{normal}(s, \mu, \sigma)$ is the likelihood that sample $s$ is obtained in a normal distribution with average $\mu$ and standard deviation $\sigma$.

The unnormalized, combined probability that the vectorized block will have use $i$ is $u p_{i}=p d_{i} * p u_{i} * p s_{i}$. The normalized probability is $p_{i}$ :

$$
p_{i}=\frac{u p_{i}}{\sum_{i} u p_{i}}
$$

The corresponding cumulative probability of use $i c_{i}$ is

$$
c_{i}=\sum_{i=0}^{i} p_{i}
$$


To assign land use to a vectorized parcel, we first decide whether the parcel should be developed at all. Because undeveloped regions in the simulated block do not have welldefined parcels, distance and size have little meaning, and therefore we cannot make use of the probabilities $p d_{i}$ and $p s_{i}$, with $i$ referring to the "undeveloped use." We therefore use only the proportion-based probability $p u_{i}$. If a random value is less than $p u_{i}$, we mark the vectorized parcel as undeveloped. Otherwise, the parcel needs to be developed so we choose a random value between $[0,1]$, and choose its land use based on the intervals $\left[0, c_{i}, . ., 1\right]$, where $i$ refers to any type of developed use. We show an example of land use assignment in Figure 3.17.

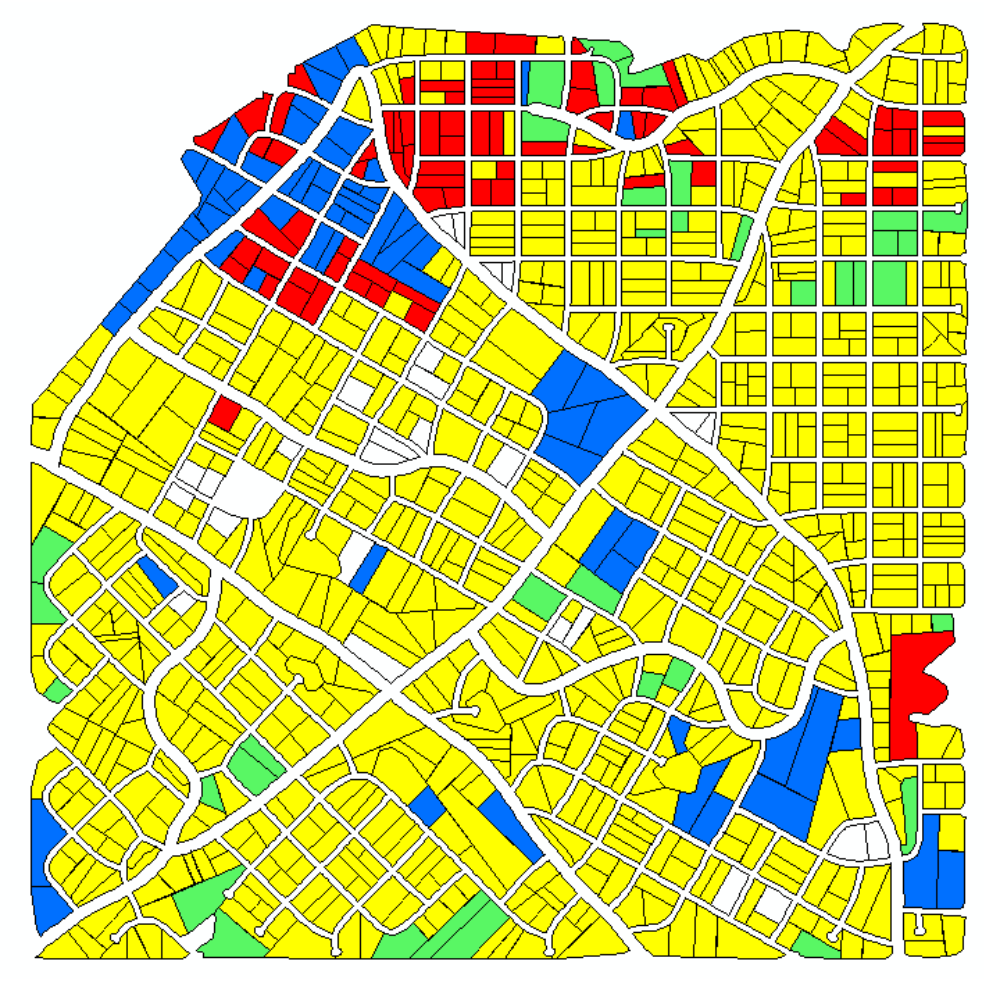

Figure 3.17: Example of land use assignment: (yellow = residential, blue = industrial, red $=$ commercial, green $=$ park)

\section{Scoring of Results}

Our generate-and-test paradigm for assigning uses to vectorized blocks requires a method for scoring the land uses assigned to each new block. We form the distance-, 
proportion-, and size-based cost terms into an additive cost function as follows:

$$
\begin{aligned}
a_{i} & =\text { area of the nearest simulation parcel of the same type } \\
c u_{i} & =\operatorname{abs}\left(u s_{i}-u_{i}\right) \\
c d_{i} & =\sum_{j}^{\text {all parcels }} d_{i j} / B B D \\
c s_{i} & =\sum_{j}^{\text {all parcels }} a b s\left(a_{j}-a_{i j}\right)
\end{aligned}
$$

where $\mathrm{BBD}$ is the length of the bounding box diagonal. Our cost function is cost $=c u_{i}+$ $c d_{i}+c s_{i}$.

If this score is less than the previous lowest score (or it is our first score), we save the subdivision, land use assignment, and new score and continue iterating. We currently repeat this process 50 times.

\section{Population Assignment}

We now assign populations (and as a side effect, usage densities) to vectorized blocks and parcels. This process distributes all of the population in the simulated block to the single, matching vectorized block. We begin by constructing a one-to-one mapping between the vectorized and simulated parcels. The mapping is segmented by land use type. For each type, the number of vectorized parcels may be greater than (GT), equal to, or less than (LT) the number of parcels in the simulated block. We handle the GT case by iteratively grouping the two smallest vectorized parcels until the number of simulated and (possibly grouped) vectorized parcels is identical. (This grouping affects only population assignment, it does not in fact merge the vectorized parcels). We handle the LT case by producing a mapping between the vectorized parcels and a subset of the simulated parcels, and distributing the population not in this subset outside of the mapping, as described below. To produce the mapping, we link each vectorized parcel to the nearest unmapped simulated parcel.

We assign the population in each simulated parcel to its corresponding vectorized parcel. If the vectorized parcel is in fact a group, we divide the simulated parcel's population across the grouped parcels in proportion to their area. If the simulated parcel was not in 
the mapped subset, we distribute the simulated parcel's population across the vectorized parcels of the same usage type, in proportion to their area. It is possible that there are in fact no vectorized parcels of the same type; this can occur when only a very small proportion of the simulated block was dedicated to this use. In this case, we ignore this usage type: we do not distribute the simulated block's population for this use to the vectorized block. Figure 3.18 shows an example of a generated density map using population assignment.

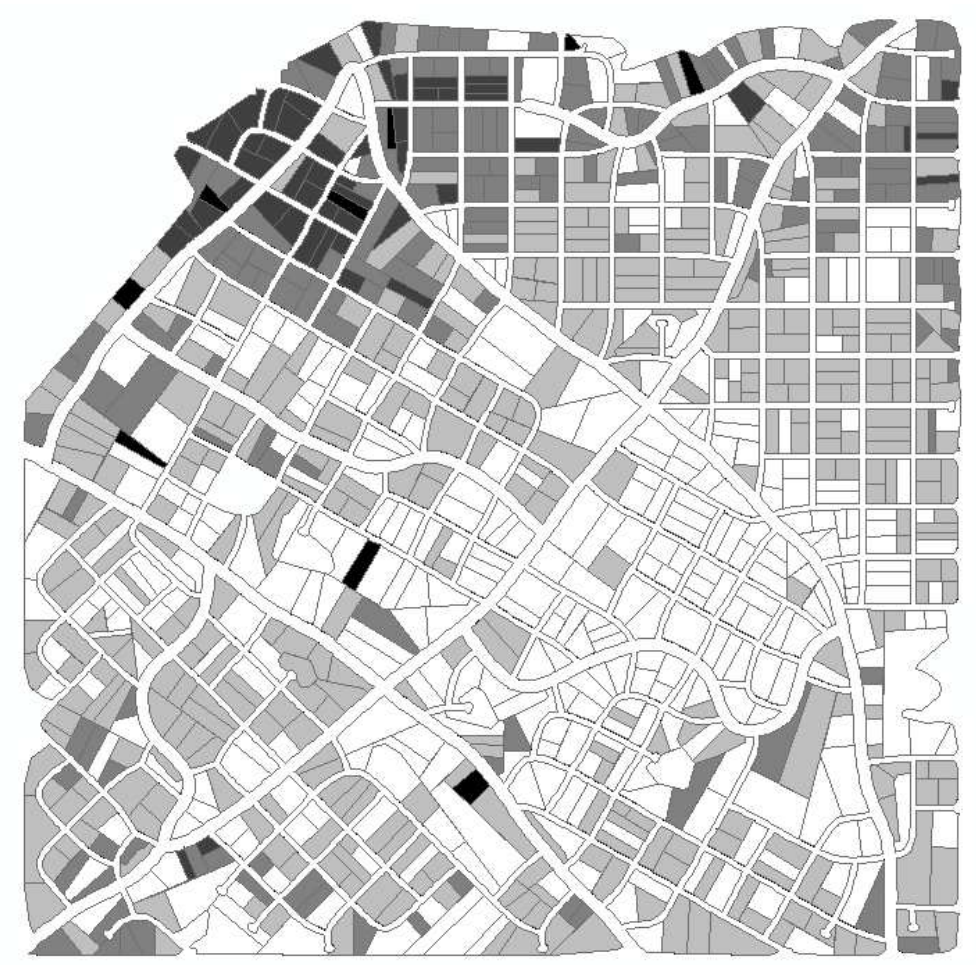

Figure 3.18: Example of density (dark is higher density) 


\section{Chapter 4}

\section{Implementation}

We implemented our algorithms in the Java language, using the open-source FengGUI library for user interfaces. FengGUI renders its widgets using OpenGL, allowing easy user control during OpenGL rendering. We used the JOGL libraries to interface between Java and OpenGL. The implementation was targeted at Windows, but should run on Linux or any other platform that JOGL supports (Linux, MacOS, Solaris).

We export program output into the ESRI Shapefile format. Exported output includes all information excepting roads. (A future version of our tool might generate road centerlines from our road graph.) We used GeoTools, an open source (LGPL) Java code library, for shapefile writing. 


\section{Chapter 5}

\section{Results}

The vectorization process proved to be a successful way of transforming the raster data from the simulation. Here we discuss the shortfalls of the current implementation and lay out the path for further research.

\subsubsection{Shortfalls}

Our program is a prototype, and still suffers from some bugs, inefficiencies, and inadequacies. When subdividing highly irregular, large blocks of land, the constraint that all generated parcels must have a road facing edge, sometimes results in oddly shaped, unrealistic parcels. Also, in places where roads are close to each other but do not touch, we sometimes generate a block spine that divides the two roads, leaving overly thin strips of road-facing land. Small-scale, dense features may be "smoothed out" by our stochastic subdivision scheme. Fortunately this happens infrequently, and is difficult to notice. Finally, at some intersections, only one of the crossing roads is straight while others have a small kink.

\subsubsection{Moving Forward}

Future work might focus on integrating the $2 \mathrm{D}$ maps we produce with other $3 \mathrm{D}$ procedural modeling tools such as CityEngine to create a complete urban model. 


\subsubsection{Examples of Vectorization}

Following are images documenting the vectorization of three unique data sets. First we show the raw data from the simulation, the land use and density maps follow. In the land use maps, clear blocks are undeveloped, yellow are residential, blue are industrial, red are commercial, and green are parks. In the density maps, the darker areas indicate higher density. The density measure is based on the ratio of population to parcel area. Note the realistic reduction of density in residential neighborhoods. 


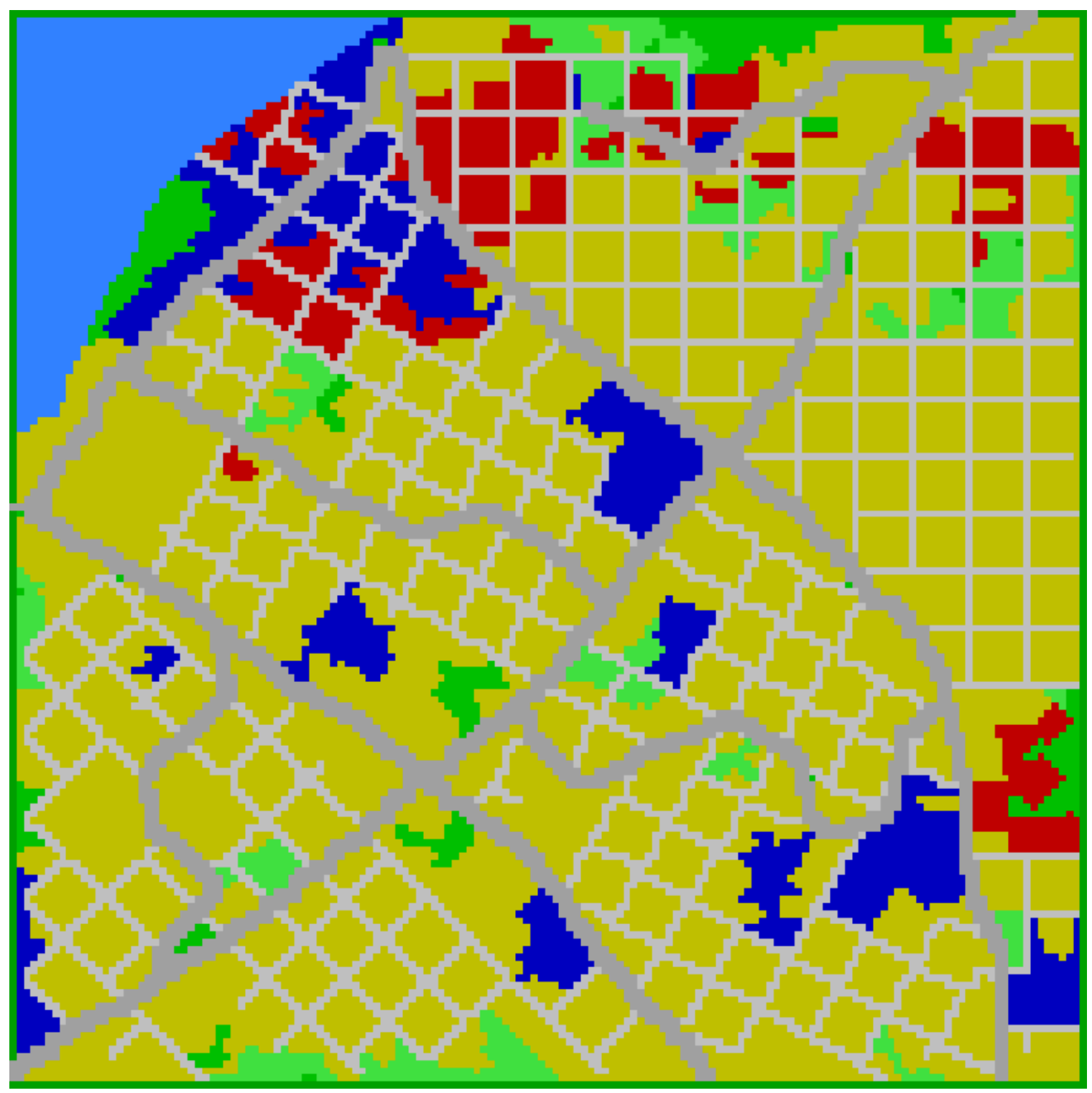

Figure 5.1: Raw simulation data: Data set 1 


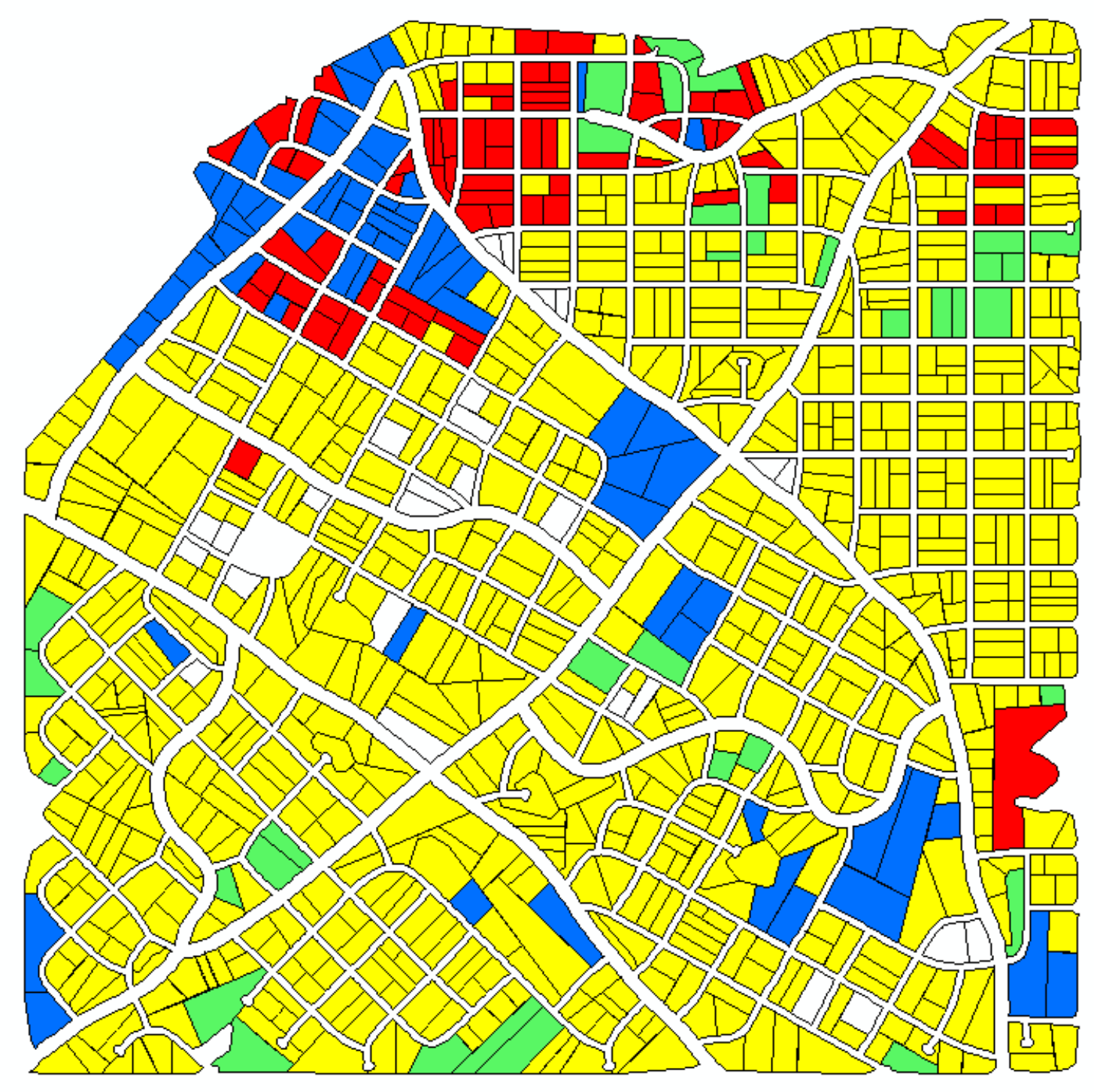

Figure 5.2: Land use assignments: Data set 1 


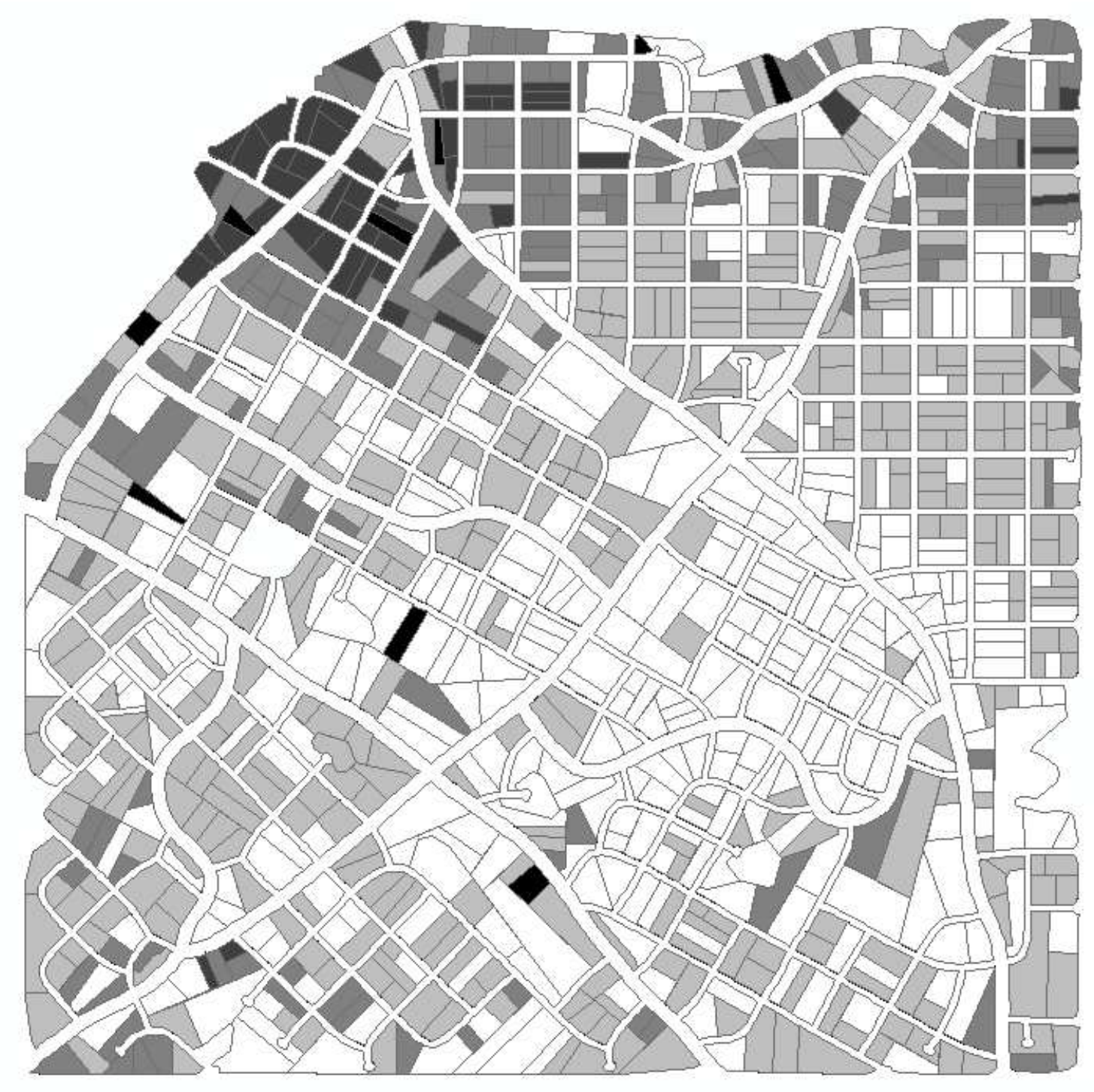

Figure 5.3: Population density visualization: Data set 1 


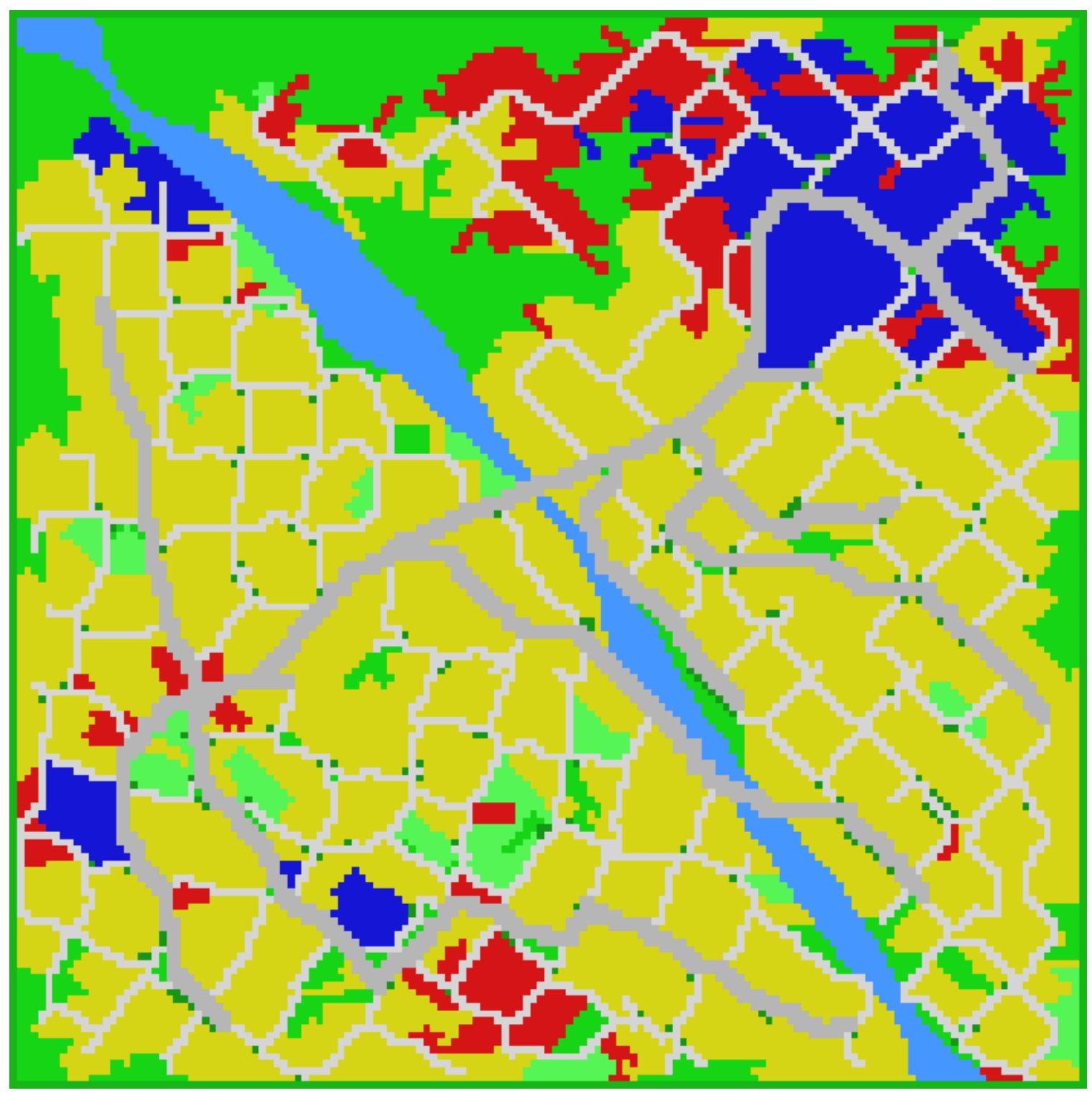

Figure 5.4: Raw simulation data: Data set 2 


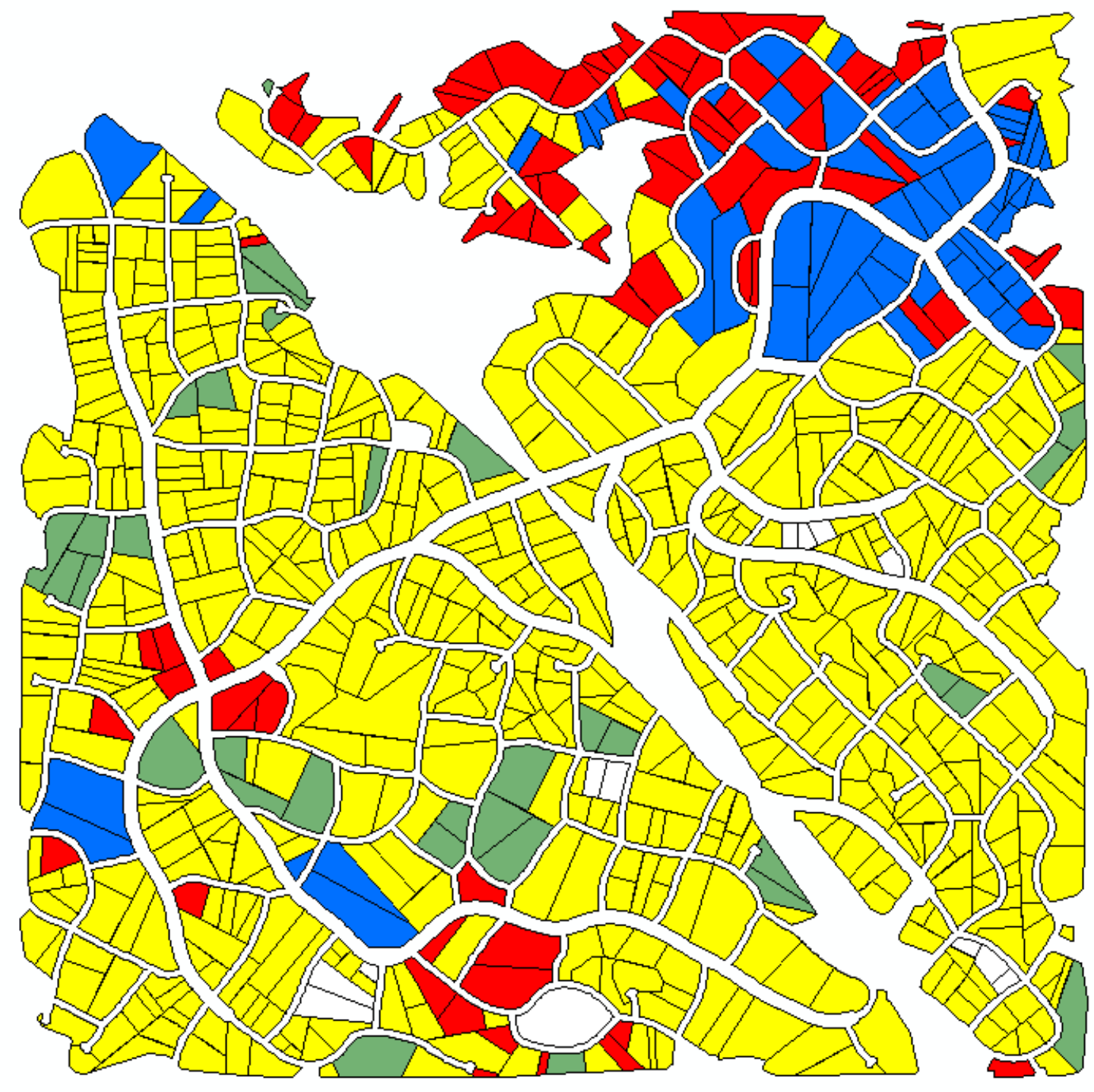

Figure 5.5: Land use assignments: Data set 2 


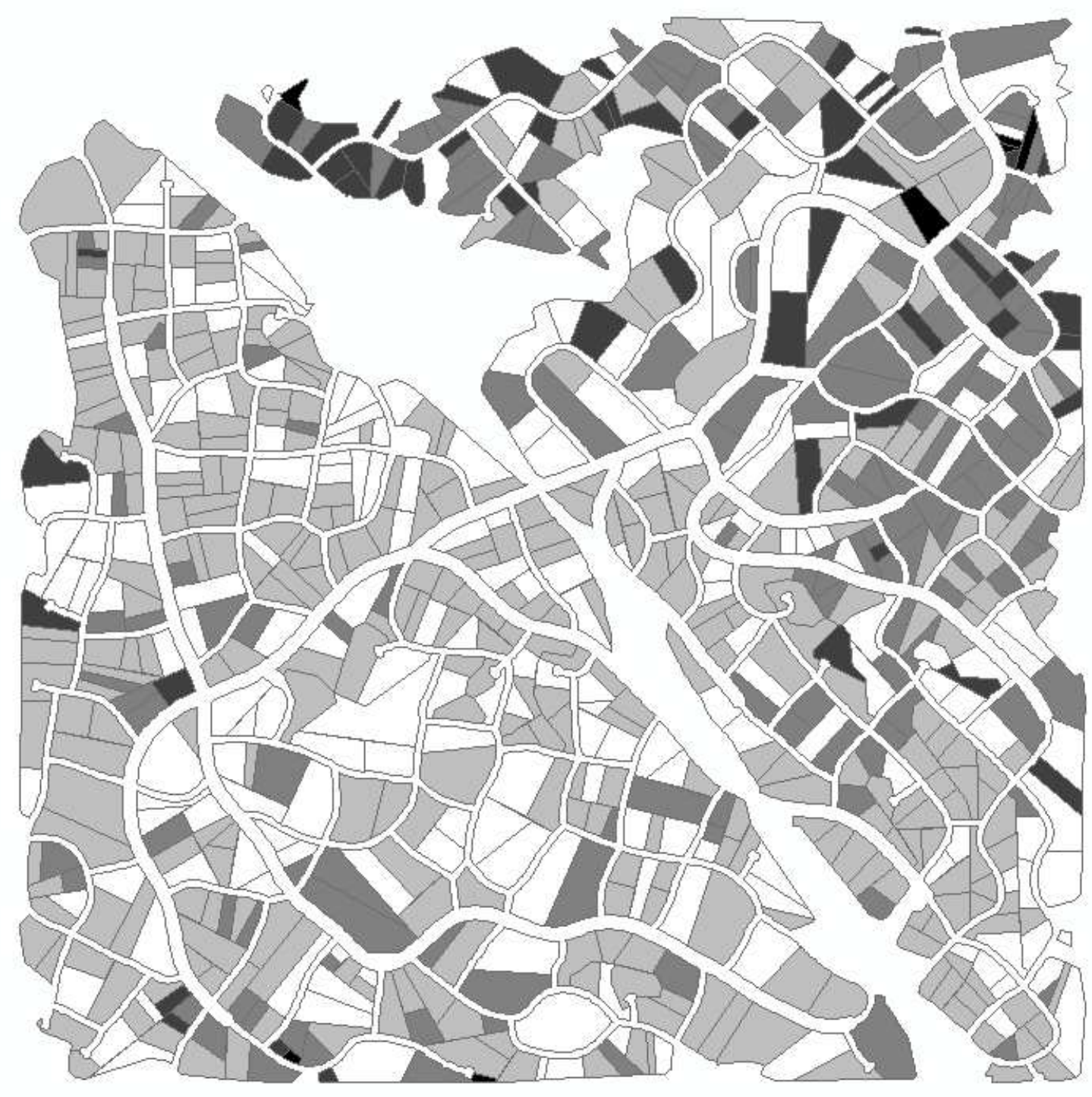

Figure 5.6: Population density visualization: Data set 2 


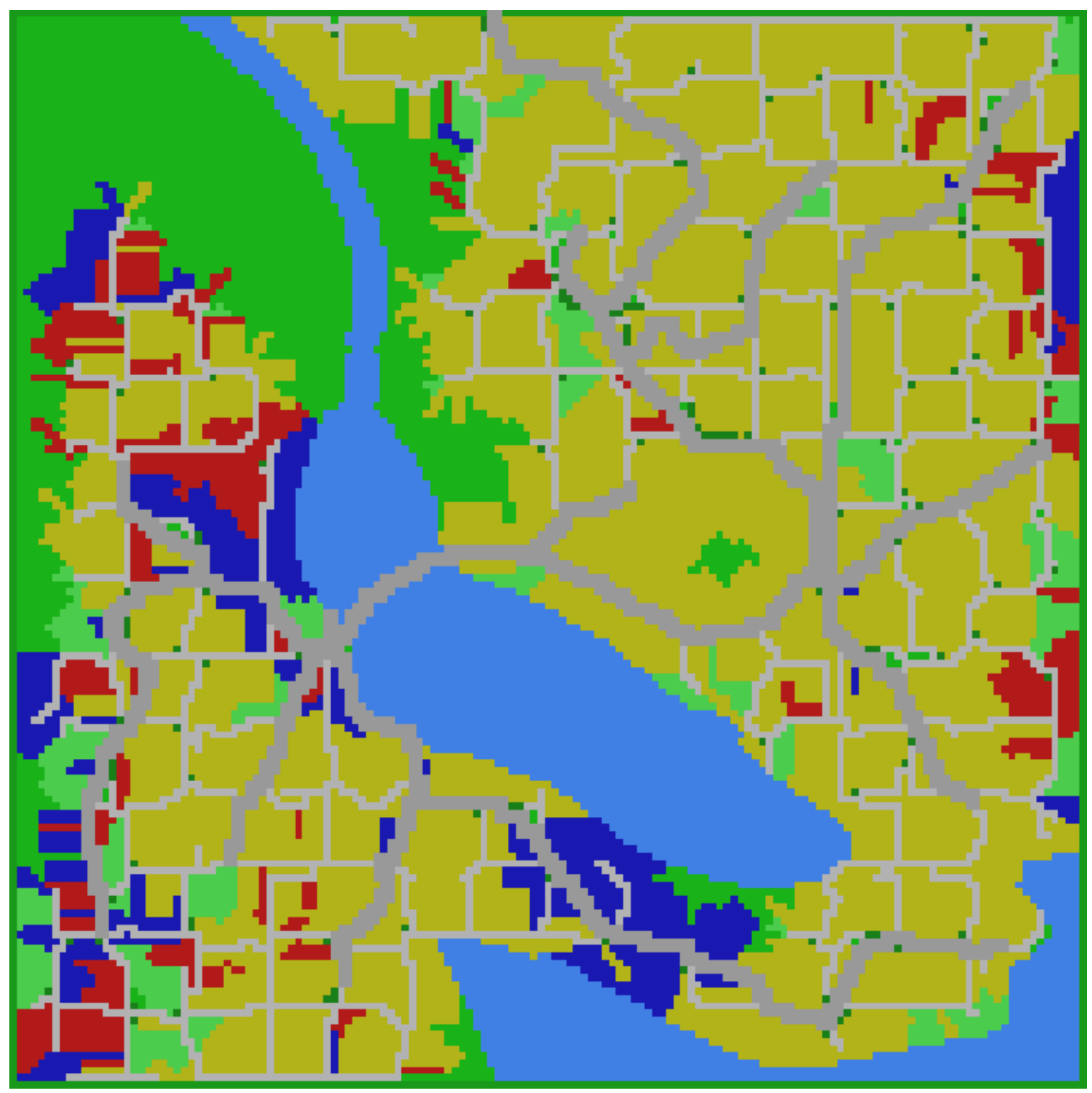

Figure 5.7: Raw simulation data: Data set 3 


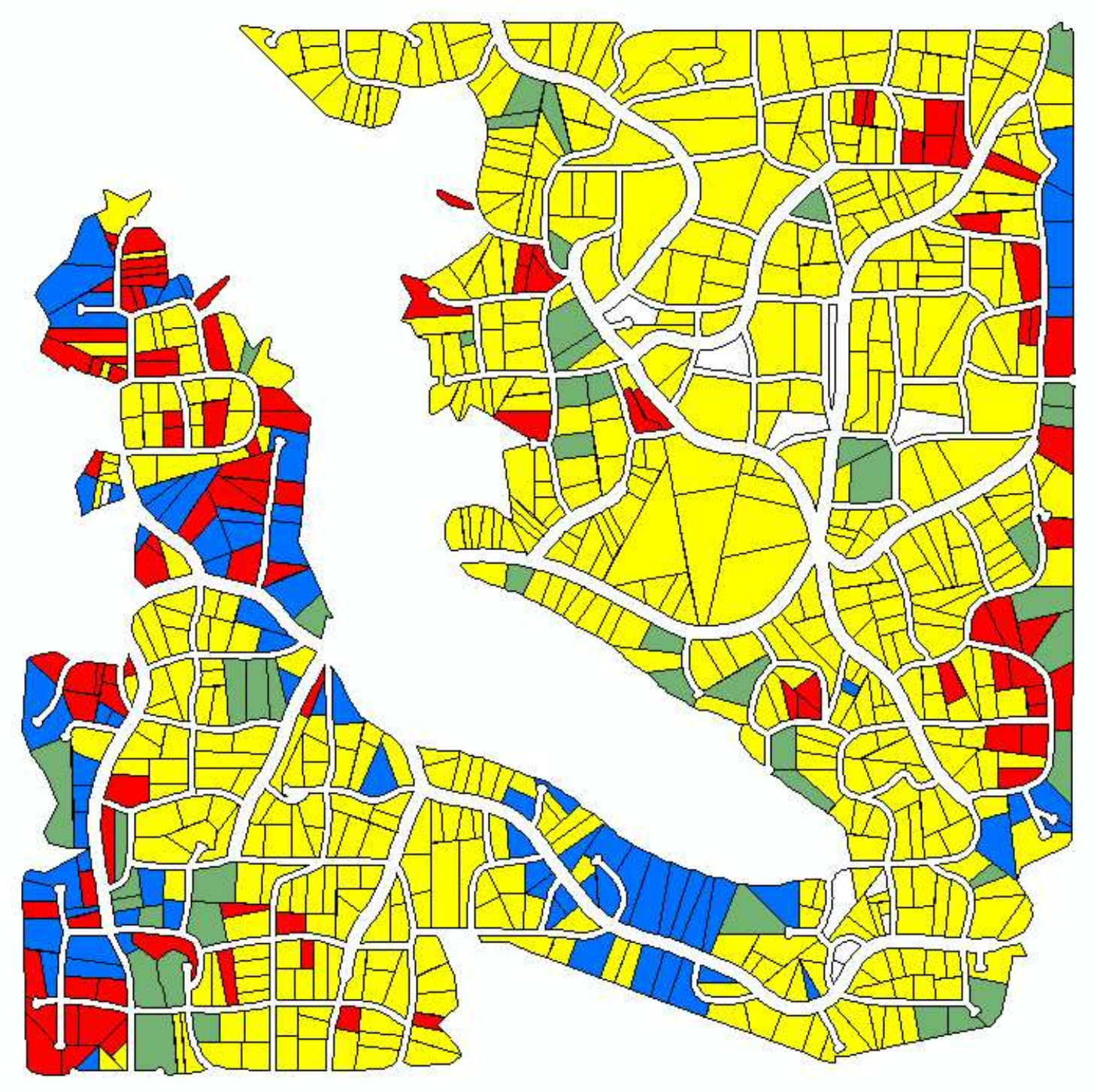

Figure 5.8: Land use assignments: Data set 3 


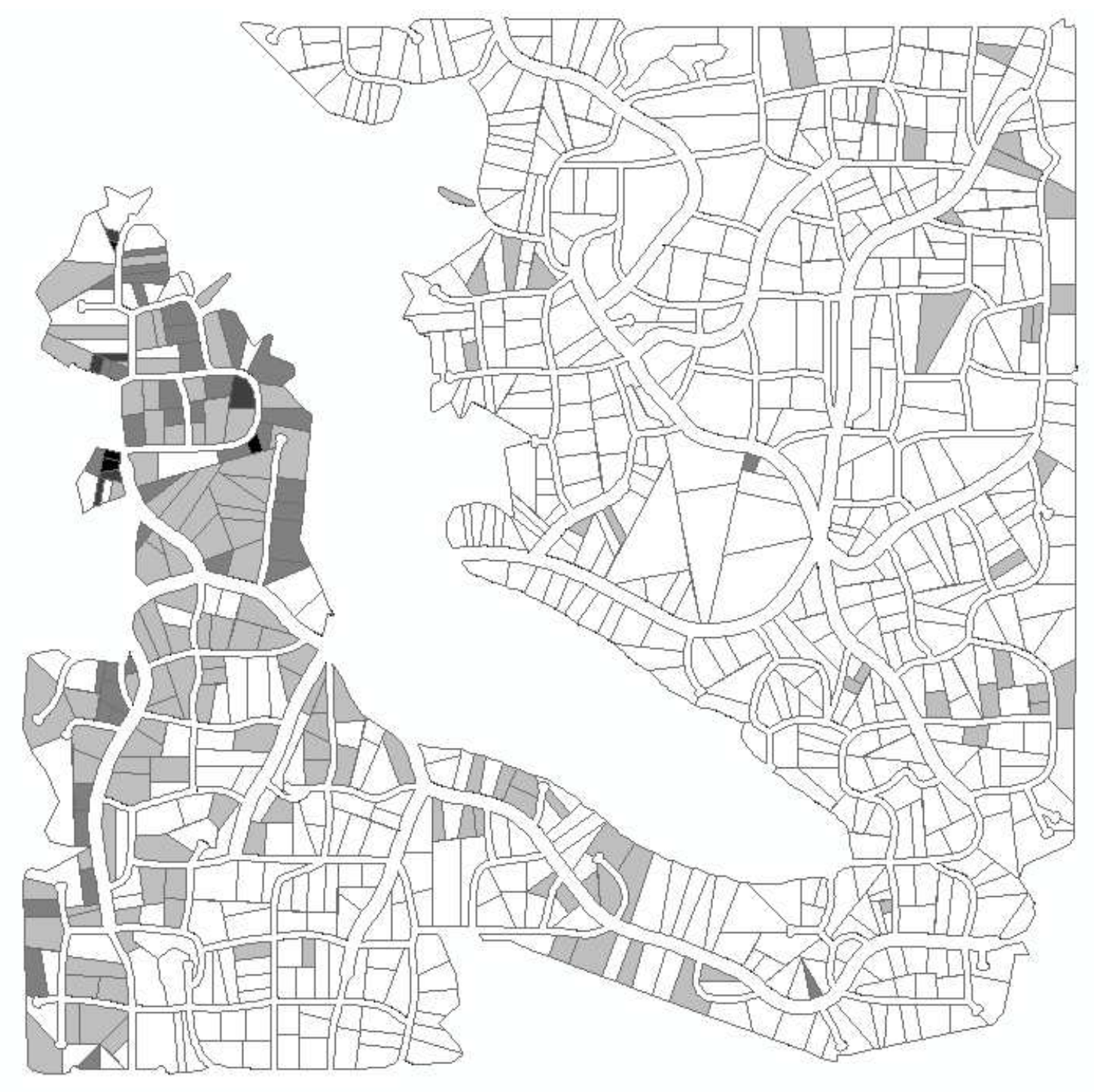

Figure 5.9: Population density visualization: Data set 3 


\section{Bibliography}

[1] Michael Ashikhmin. Synthesizing natural textures. In I3D '01: Proceedings of the 2001 symposium on Interactive 3D graphics, pages 217-226, New York, NY, USA, 2001. ACM Press.

[2] Daniel Bekins and Daniel G. Aliaga. Build-by-number: Rearranging the real world to visualize novel architectural spaces. volume 00, pages 143-150. IEEE Computer Society, October 23-28 2005.

[3] Pravin Bhat, Stephen Ingram, and Greg Turk. Geometric texture synthesis by example. In SGP '04: Proceedings of the 2004 Eurographics/ACM SIGGRAPH symposium on Geometry processing, pages 41-44, New York, NY, USA, 2004. ACM Press.

[4] Ching-Chien Chen, Craig A. Knoblock, Cyrus Shahabi, Yao-Yi Chiang, and Snehal Thakkar. Automatically and accurately conflating orthoimagery and street maps. In GIS '04: Proceedings of the 12th annual ACM international workshop on Geographic information systems, pages 47-56, New York, NY, USA, 2004. ACM Press.

[5] Yanyun Chen, Lin Xia, Tien-Tsin Wong, Xin Tong, Hujun Bao, Baining Guo, and Heung-Yeung Shum. Visual simulation of weathering by y-ton tracing. In SIGGRAPH '05: ACM SIGGRAPH 2005 Papers, pages 1127-1133, New York, NY, USA, 2005. ACM Press.

[6] Yao-Yi Chiang, Craig A. Knoblock, and Ching-Chien Chen. Automatic extraction of road intersections from raster maps. In GIS '05: Proceedings of the 13th annual ACM international workshop on Geographic information systems, pages 267-276, New York, NY, USA, 2005. ACM Press.

[7] Barbara Cutler, Julie Dorsey, Leonard McMillan, Matthias Müller, and Robert Jagnow. 
A procedural approach to authoring solid models. In SIGGRAPH '02: Proceedings of the 29th annual conference on Computer graphics and interactive techniques, pages 302-311, New York, NY, USA, 2002. ACM Press.

[8] Dov Dori and Wenyin Liu. Sparse pixel vectorization: An algorithm and its performance evaluation. IEEE Transactions on Pattern Analysis and Machine Intelligence, 21(3):202-215, 1999.

[9] Julie Dorsey, Alan Edelman, Henrik Wann Jensen, Justin Legakis, and Hans Kohling Pedersen. Modeling and rendering of weathered stone. In SIGGRAPH '99: Proceedings of the 26th annual conference on Computer graphics and interactive techniques, pages 225-234, New York, NY, USA, 1999. ACM Press/Addison-Wesley Publishing Co.

[10] David S. Ebert, F. Kenton Musgrave, Darwyn Peachey, Ken Perlin, and Steven Worley. Texturing and Modeling: A Procedural Approach. Morgan Kaufmann Publishers Inc., San Francisco, CA, USA, 2002.

[11] Alexei A. Efros and William T. Freeman. Image quilting for texture synthesis and transfer. In SIGGRAPH '01: Proceedings of the 28th annual conference on Computer graphics and interactive techniques, pages 341-346, New York, NY, USA, 2001. ACM Press.

[12] Kuo-Chin Fan, Den-Fong Chen, and Ming-Gang Wen. A new vectorization-based approach to the skeletonization of binary images. ICDAR, 02:627, 1995.

[13] Alain Fournier, Don Fussell, and Loren Carpenter. Computer rendering of stochastic models. Commun. ACM, 25(6):371-384, 1982.

[14] Stefan Greuter, Jeremy Parker, Nigel Stewart, and Geoff Leach. Real-time procedural generation of 'pseudo infinite' cities. In GRAPHITE '03: Proceedings of the 1st international conference on Computer graphics and interactive techniques in Australasia and South East Asia, pages 87-ff, New York, NY, USA, 2003. ACM Press.

[15] Sotirios Gyftakis, Peggy Agouris, and Anthony Stefanidis. Image-based change detection of areal objects using differential snakes. In GIS '05: Proceedings of the 13th annual ACM international workshop on Geographic information systems, pages 135-142, New York, NY, USA, 2005. ACM Press. 
[16] Evan Hahn, Prosenjit Bose, and Anthony Whitehead. Persistent realtime building interior generation. In sandbox '06: Proceedings of the 2006 ACM SIGGRAPH symposium on Videogames, pages 179-186, New York, NY, USA, 2006. ACM Press.

[17] Mikako Harada, Andrew Witkin, and David Baraff. Interactive physically-based manipulation of discrete/continuous models. In SIGGRAPH '95: Proceedings of the 22nd annual conference on Computer graphics and interactive techniques, pages 199-208, New York, NY, USA, 1995. ACM Press.

[18] Sven Havemann. Generative Mesh Modeling. PhD thesis, TU Braunschweig, 2005.

[19] Johannes Kopf, Chi-Wing Fu, Daniel Cohen-Or, Oliver Deussen, Dani Lischinski, and Tien-Tsin Wong. Solid texture synthesis from 2d exemplars. In SIGGRAPH '0\%: ACM SIGGRAPH 2007 papers, page 2, New York, NY, USA, 2007. ACM Press.

[20] Ares Lagae, Oliver Dumont, and Philip Dutré. Geometry synthesis by example. In International Conference on Shape Modeling and Applications, pages 176-185. IEEE Computer Society, 2005.

[21] Justin Legakis, Julie Dorsey, and Steven Gortler. Feature-based cellular texturing for architectural models. In SIGGRAPH '01: Proceedings of the 28th annual conference on Computer graphics and interactive techniques, pages 309-316, New York, NY, USA, 2001. ACM Press.

[22] John-Peter Lewis. Texture synthesis for digital painting. In SIGGRAPH '84: Proceedings of the 11th annual conference on Computer graphics and interactive techniques, pages 245-252, New York, NY, USA, 1984. ACM Press.

[23] Wenyin Lieu and Dov Dori. From rasters to vectors: Extracting visual information from line drawings. PAA, 2(1):10-21, 1999.

[24] J. B. Mena. Automatic vectorization of segmented road networks by geometrical and topological analysis of high resolution binary images. Know.-Based Syst., 19(8):704$718,2006$.

[25] Paul Merrell. Example-based model synthesis. In I3D '07: Proceedings of the 2007 symposium on Interactive 3D graphics and games, pages 105-112, New York, NY, USA, 2007. ACM Press. 
[26] Pascal Müller, Peter Wonka, Simon Haegler, Andreas Ulmer, and Luc Van Gool. Procedural modeling of buildings. 25(3):614-623, 2006.

[27] Pascal Müller, Gang Zeng, Peter Wonka, and Luc Van Gool. Image-based procedural modeling of facades. In SIGGRAPH '0\%: ACM SIGGRAPH 2007 papers, page 85, New York, NY, USA, 2007. ACM Press.

[28] F. K. Musgrave, C. E. Kolb, and R. S. Mace. The synthesis and rendering of eroded fractal terrains. In SIGGRAPH '89: Proceedings of the 16th annual conference on Computer graphics and interactive techniques, pages 41-50, New York, NY, USA, 1989. ACM Press.

[29] Yoav I. H. Parish and Pascal Müller. Procedural modeling of cities. In Eugene Fiume, editor, Proceedings of ACM SIGGRAPH 2001, pages 301-308, New York, NY, USA, 2001. ACM Press.

[30] Ken Perlin. An image synthesizer. In SIGGRAPH '85: Proceedings of the 12th annual conference on Computer graphics and interactive techniques, pages 287-296, New York, NY, USA, 1985. ACM Press.

[31] Ken Perlin and Luiz Velho. Live paint: painting with procedural multiscale textures. In SIGGRAPH '95: Proceedings of the 22nd annual conference on Computer graphics and interactive techniques, pages 153-160, New York, NY, USA, 1995. ACM Press.

[32] Robert Pless and David Jurgens. Road extraction from motion cues in aerial video. In GIS '04: Proceedings of the 12th annual ACM international workshop on Geographic information systems, pages 31-38, New York, NY, USA, 2004. ACM Press.

[33] Helmut Pottmann, Yang, Johannes Wallner, Alexander Bobenko, and Wenping Wang. Geometry of multi-layer freeform structures for architecture. In SIGGRAPH '0\%: ACM SIGGRAPH 2007 papers, page 65, New York, NY, USA, 2007. ACM Press.

[34] Przemyslaw Prusinkiewicz, Aristid Lindenmayer, and James Hanan. Development models of herbaceous plants for computer imagery purposes. In SIGGRAPH '88: Proceedings of the 15th annual conference on Computer graphics and interactive techniques, pages 141-150, New York, NY, USA, 1988. ACM Press. 
[35] William T. Reeves. Particle systems-a technique for modeling a class of fuzzy objects. In SIGGRAPH '83: Proceedings of the 10th annual conference on Computer graphics and interactive techniques, pages 359-375, New York, NY, USA, 1983. ACM Press.

[36] Craig W. Reynolds. Flocks, herds and schools: A distributed behavioral model. In SIGGRAPH '87: Proceedings of the 14th annual conference on Computer graphics and interactive techniques, pages 25-34, New York, NY, USA, 1987. ACM Press.

[37] Karl Sims. Evolving virtual creatures. In SIGGRAPH '94: Proceedings of the 21st annual conference on Computer graphics and interactive techniques, pages 15-22, New York, NY, USA, 1994. ACM Press.

[38] Jeffrey Smith, Jessica Hodgins, Irving Oppenheim, and Andrew Witkin. Creating models of truss structures with optimization. In SIGGRAPH '02: Proceedings of the 29th annual conference on Computer graphics and interactive techniques, pages 295301, New York, NY, USA, 2002. ACM Press.

[39] George Stiny and W. J. Mitchell. The palladian grammar. Environment and Planning $B, 5(1): 5-18,1978$.

[40] Gwenola Thomas and Stéphane Donikian. Modelling virtual cities dedicated to behavioural animation. Computer Graphics Forum, 19(3):71-80, 2000.

[41] Li-Yi Wei and Marc Levoy. Fast texture synthesis using tree-structured vector quantization. In SIGGRAPH '00: Proceedings of the 27th annual conference on Computer graphics and interactive techniques, pages 479-488, New York, NY, USA, 2000. ACM Press/Addison-Wesley Publishing Co.

[42] Chris White. King kong: the building of 1933 new york city. In SIGGRAPH '06: ACM SIGGRAPH 2006 Sketches, page 96, New York, NY, USA, 2006. ACM Press.

[43] Peter Wonka, Michael Wimmer, François Sillion, and William Ribarsky. Instant architecture. In SIGGRAPH '03: ACM SIGGRAPH 2003 Papers, pages 669-677, New York, NY, USA, 2003. ACM Press.

[44] T. Y. Zhang and C. Y. Suen. A fast parallel algorithm for thinning digital patterns. Commun. ACM, 27(3):236-239, 1984. 
[45] Howard Zhou, Jie Sun, Greg Turk, and James M. Rehg. Terrain synthesis from digital elevation models. IEEE Transactions on Visualization and Computer Graphics, 13(4):834-848, July/August 2007.

[46] Ju Jia Zou and Hong Yan. Cartoon image vectorization based on shape subdivision. cgi, 00:0225, 2001. 